\title{
Total Dual Integrality from Directed Graphs, Crossing Families, and Sub- and Supermodular Fanctions
}

\author{
A. Schrijver \\ Instituut voor Actuariaat en Econometrie \\ Universiteit van Ansterdam \\ Jodenbreestraat 23 \\ 1011 NH Amsterdam, Holland
}

\begin{abstract}
We review old and describe some new results asserting the total dual integrality of certain systems of inequalities involving directed graphs, crossing families, and sub- and supermodular functions. Moreover, we discuss their interrelations.
\end{abstract}

\section{Introduction}

During the last few years several models have been proposed to prove integer optimum solutions for certain linear programs. The fundamental model of (poly)matroids and their intersections described by Edmonds, was followed by more sophisticated frameworks designed by Johnson (cut-set polyhedra), Edmonds and Giles, Hoffman and Schwartz (lattice polyhedra), Frank (kernel systems, generalized polymatroids), Hassin, Lawler and Martel (polymatroidal network flows). The integrality of the optima in question imply combinatorial min-max relations, containing as special cases Konig's matching theorem, the max-flow min-cut theorem, Fulkerson's optimum arborescence theorem, the Lucchesi-Younger theorem, Nash-Williams' orientation theorem.

These models have in common that they involve functions satisfying a certain sub- or supermodular law. This allows the proof technique (set up by Edmonds, Giles, Hoffman, Johnson, Lovász, Robertson) of showing first that the active constraints in the optimum of the linear program can be chosen to be "nice" (e.g., "cross-free" or "laminar"), and next that nice constraint sets are totally unimodular. By Hoffman and Kruskal's result this yields integer optimum primal solutions if the right hand sides are integer, and integer optimum dual solutions if the objective function is integer. 
More precisely, these models assert, or contain as special case, that any linear program with integer objective function over one of the constraint sets

$$
x\left(8_{A}^{-}\left(V^{\prime}\right)\right)-x\left(8_{A}^{+}\left(V^{\prime}\right)\right) \leq f\left(V^{\prime}\right) \quad\left(V^{\prime} \in C\right)
$$

or

$$
x\left(8_{A}^{-}\left(V^{\prime}\right)\right) \leq f\left(V^{\prime}\right) \quad\left(V^{\prime} \in C\right)
$$

has an integer optimum dual solution, for certain directed graphs $D=(V, A)$, collections $C$ of subsets of $V$ and functions $f: C \rightarrow R$. [Here $x$ is a vector in $\mathbb{R}^{A}, 8_{A}^{-}\left(V^{\prime}\right)$ and $\delta_{A}^{+}\left(V^{\prime}\right)$ denote the sets of arcs entering and leaving $V^{\prime}$, respectively, and $x\left(A^{\prime}\right):=\sum_{a \in A^{\prime}} x(a)$ for $A^{\prime} \subseteq A$.] That is, the system (1) and (2) is totally dual integral (see below). Hence, by a result of Hoffman and Edmonds and Giles, also the primal program over (1) or (2) has an integer optimum solution if $f$ is integer.

A primal linear program over (1) and (2) has as typical optimum an object of "connector type", e.g., a path, chain, arborescence, matching, or covering. The dual solution corresponds to an optimum packing or covering with "cut type" objects, like cuts, antichains, stars.

In Section 2 we give a survey of old and new total dual integrality results of this type, and we describe their interrelations. Starting with the basic results on bipartite matching and network flows, via the Lucchesi-Younger theorem and the matroid intersection theorem, we pass to the more general models. At the moment the most general model scems to be Grishuhin's, which has as an axiom that "nice" constraint sets are totally unimodular. So half of the proof technique described above is now put in an axiom, which seems, however, in general difficult to check (continuing in this direction the most general model would just consist of the definition of total dual integrality). We describe a model, weaker than Grishuhin's, but with more easily verifiable axioms, which also contains all other models.

The interrelations between the several models we describe below have the form of "direct constructions", which give that one class of inequality systems is included in another class of inequality systems, or may be derived from it by adding some redundant inequalities (redundant also with respect to total dual integrality). These constructions also yield that any algorithm for the one problem directly passes over to an algorithm for the other problem. Some of the constructions are (well-)known, like the one reducing network flows to bipartite matching, some other of them seem to be new. 
There is a "polar" type of results, in the sense that Konig's edgecolouring theorem is polar to Konig's matching theorem, and that Edmonds' disjoint arborescence theorem is polar to Fulkerson's optimum arborescence theorem. Essentially it means interchanging "connector type" and "cut type" objects. Typically, these polar results assert that for a given digraph $D=(V, A)$ and collection $C$ of subsets of $V$, if each $V^{\prime}$ in $C$ is entered by at least $k$ arcs of $D$, then the arcs can be coloured with $k$ colours such that each $V^{\prime}$ in $C$ is entered by at least one arc of each colour (similarly, if we replace "at least" by "at most"). The question is, for which digraphs $D$ and collections $C$ is this true?

In Section 3 we review this type of results, including Menger's theorem, Konig's edge-colouring theorem, Edmonds' disjoint arborescence theorem, Frank's Kernel system theorem, and a new theorem of this type. We also comment on how to include sub- or supermodularity here.

In this paper we neglect the important algorithmic aspects of the models. To this end we refer to the papers of Cunningham and Frank [1982], Edmonds [1967, 1970, 1979], Frank [1980, 1980a, 1981, 1981a, 1981c, 1982], Fujishige [1978], Hassin [1978], Karzanov [1979], Lawler [1975], Lawler and Martel [1982a], Lovász [1976], Lucchesi [1976], Schonsleben [1980].

For most of the models the polynomial solvability of the corresponding optimization problems can be derived with the ellipsoid method. However, there is the problem of how to represent the problems: e.g., an explicit list of all sets in the collection C, together with their value under $f$, would take generally exponential space. In Section 4 we make some observations on how to represent "crossing" families in space polynomially bounded by the underlying set (and on a similar representation for certain perfect graphs). The function $f$, however, generally needs to be given by an oracle, as there may exist much more than exponentially many sub- or supermodular functions.

TOTAL DUAL INTEGRALITY. Here we review some of the theory of total dual integrality. By definition, a system $A x \leq b$ of inequalities is called totally dual integral or t.d.i. if the minimum in the linear programming duality equation

$$
\max \{w x \mid A x \leq b\}=\min \{y b \mid y \geq 0, y A=w\}
$$

has an integer optimum solution for each integer objective function $w$ for which the minimum exists. Hoffman [1974] and Edmonds and Giles [1977] showed that if $A x \leq b$ is t.d.i. and $b$ is integer then also the maximum in (3) has an integer optimum solution. 
The system $A x \leq b$ is called box totally dual integral or box t.d.i. if the system " $A x \leq b, c \leq x \leq d$ " is t.d.i. for all vectors $c$, $d$. It is not difficult to see that box total dual integrality implies total dual integrality.

Note that (box) total dual integrality is a property of systems of inequalities, and not only of polyhedra. Generally, a t.d.i. system must contain more constraints then necessary for just defining the polyhedron.

Giles and Pulleyblank [1979] showed that each polyhedron with integer vertices can be described by a t.d.i. system with integer right hand sides. In Schrijuer [1981] it is shown that each full-dimensional rational polyhedron is described by a unique minimal t.d.i. system (minimal with respect to removing constraints), and that each face of the polyhedron contains integer points iff the right hand sides of this minimal system are integer.

Edmonds and Giles [1977] announced that each minimal box t.d.i. system for a full-dimensional polyhedron has $0,+1,-1$ left hand sides. Note that each minimal box t.d.i. system is also minimal t.d.i. Hence a t.d.i. system which is not box t.d.i. cannot be made box t.d.i by adding redundant constraints.

There are some straightforward further observations on total dual integrality, which we will use tacitly in the sequel. If $A_{1} x \leq b_{1}$ and $A_{2} x \leq b_{2}$ determine the same polyhedron, and each inequality in $A_{1} x \leq b_{1}$ is a nonnegative integer combination of inequalities in $A_{2} x \leq b_{2}$, then $A_{1} x \leq b_{1}$ (box) t.d.i. implies $A_{2} x \leq b_{2}$ (box) t.d.i.

If $A x \leq b$ is an inequality system, and the vector $x$ is extended by a new coordinate, say $r$, and if $c$ is an integer vector and $d$ is a number, then $A x \leq b$ is (box) t.d.i. if and only if the system "Ax $\leq b, c x+r=d$ " is (box) t.d.i. This yield that if a coordinate occurs with coefficient 1 or -1 in a valid equation, we can eliminate this coordinate.

If the system " $A x \leq b, c x \leq d$ " is (box) t.d.i., then the system " $A x \leq b, c x=d$ " is (box) t.d.i. If $A x \leq b$ is (box) t.d.i. and $c$ is a vector, then the system $A x \leq b+A c$ is (box) t.d.i. So (box) total dual integrality is stable under translations.

SOME TERMINOLOGY AND NOTATION. If $D=(V, A)$ is a directed graph and $V^{\prime} \subseteq V$, then $8^{+}\left(V^{\prime}\right)$ or $8_{A}^{+}\left(V^{\prime}\right)$ denotes the set of arcs of $D$ leaving $V^{\prime}$. Similarly, $8^{-}\left(V^{\prime}\right)$ or $8_{A}^{-}\left(V^{\prime}\right)$ denotes the set of ares of $D$ entering $V^{\prime}$.

If $S$ is a set and $x: S \rightarrow R$, then by definition

$$
x\left(S^{\prime}\right):=\Sigma_{s \in s^{\prime}} x(s)
$$


for $S^{\prime} \subseteq S$.

Two subsets $S^{\prime}$ and $S^{\prime \prime}$ of the set $S$ are called intersecting if $S^{\prime} \cap S^{\prime \prime} \neq \varnothing$. They are called crossing if $S^{\prime} \cap S^{\prime \prime} \neq S$, $S^{\prime} \cup S^{\prime \prime} \neq S, S^{\prime} \nsubseteq S^{\prime \prime}$ and $S^{\prime \prime} \nsubseteq S^{\prime}$.

If $\mathbf{C}$ is a collection of subsets of $S$, and $f: \mathbf{C} \rightarrow \boldsymbol{R}$, then $\mathbf{C}$ is called a lattice family and $f$ is called submodular if for all $S^{\prime}, S^{\prime \prime}$ in $C$,

(i) $S^{\prime} \cap S^{\prime \prime}$ and $S^{\prime} \cup S^{\prime \prime}$ belong to $C$,

$$
\text { (ii) } f\left(S^{\prime}\right)+f\left(S^{\prime \prime}\right) \geq f\left(S^{\prime} \cap S^{\prime \prime}\right)+f\left(S^{\prime} \cup S^{\prime \prime}\right) \text {. }
$$

$\mathbf{C}$ is called an intersecting family and $f$ is submodular on intersecting pairs if (5) holds for all intersecting sets $S^{\prime}$ and $S^{\prime \prime}$ in C . C is called a crossing family and $f$ is submodular on crossing pairs if (5) holds for all crossing sets $S^{\prime}$ and $S^{\prime \prime}$ in $\mathrm{C}$.

If we replace in (5) (ii) the sign $\geq$ by $\leq$, the term "submodular" is replaced by supermodular. If (5) (ii) aiways holds with equality, the term modular (= submodular + supermodular) is used.

A collection $\mathbf{C}$ is called cross-free if $\mathbf{C}$ contains no crossing sets. $P(S)$ denotes the collection of all subsets of $S$.

\section{Optimum Connectors and Cut-collections, and Generalizations}

We now give a survey of results and frameworks of "EdmondsGiles" type, each of which gives (disguised or openly) the box total dual integrality of inequality systems of one of the following forms:

$$
x\left(8^{-}\left(V^{\prime}\right)\right) \leq f\left(V^{\prime}\right) \quad\left(V^{\prime} \in C\right),
$$

or

$$
x\left(\delta^{-}\left(V^{\prime}\right)\right)-x\left(8^{+}\left(V^{\prime}\right)\right) \leq f\left(V^{\prime}\right) \quad\left(V^{\prime} \in \mathbf{C}\right),
$$

for certain digraphs $D=(V, A)$, collections $C$ of subsets of $V$, and functions $f: \mathbf{C} \rightarrow \boldsymbol{R}$. The corresponding primal linear programs then ask for an optimum object of "connector type" (like a path, flow, matching, covering, arborescence), while the dual programs ask for optimum collections of objects of "cut type" (like a packing of cuts, a covering by antichains). In Section 3 we consider the "polar" problem of finding optimum cut type objects and optimum collections of connector type objects.

Starting with the simple, basic results on bipartite matching and network flows, we discuss the several schemes in some order of increasing generality and complexity, and we go into the relations between them. 
We discuss the following models and implications. More implications follow from taking the transitive closure of implications.

I. Konig's matching and covering theorems (Konig [1931, 1932]).

II. Menger's theorem and flow and circulation problems (Menger [1927], Ford and Fulkerson [1956], Elias, Feinstein and Shannon [1956]: $<=>$ I.

III. Shortest paths: $<=$ II.

IV. Partitions of partially ordered sets (Dilworth [1950], Greene and Kleitman [1976], Green [1976]): < = II.

V. Common SDR's (Ford and Fulkerson [1958]): => I.

VI. Trees and forests in undirected graphs (Edmonds [1970]).

VII. Optimum arborescences (Fulkerson [1974]): $=>$ III, VI.

VIII. Optimum bi-branchings (Schrijver [1982a]): $=>$ VII.

IX. Lucchesi-Younger theorem (Lucchesi and Younger [1978]): $=>$ I, IV.

X. Optimum strong connectors (Schrijver [1982a]): $<=>$ IX, $=>$ VIII.

XI. Orientations of undirected graphs (Nash-Williams [1969]).

XII. Matroids, polymatroids and submodular functions (Edmonds [1970,1971]): => VI.

XIII (Poly)matroid intersection (Edmonds [1970,1979]): => I,V,VII,XII.

XIV.Submodular functions on crossing families (Frank and Tardos [1981]): <=> XIII, => IX,XI.

XV. Generalized polymatroids (Frank [1981b]) $<=>$ XIV.

XVI. Blockers of common independent sets (Groflin and Hoffman [1981]): $<=$ XIV.

XVII. Kernel systems (Frank [1979a]): => VI, XII,XVI.

XVIII. Crossing directed cuts (Edmonds and Giles [1977]): $<=$ XIV, => VI, IX,XII, XVI, XVII.

XIX. Crossing families on directed graphs I: $=>$ XVIII, X.

XX. Crossing families on directed graphs II: the Edmonds-Giles scheme (Edmonds and Giles [1977]): $<=$ XIV , $=>$ XI, XV, XVI, XVIII.

XXI. Polymatroidal network flows (Hassin [1978], Lawler and Martel $[1980,1981]):<=>$ XX. 
XXII. Distributive lattices (Groflin and Hoffman [1982]): $<=>$ XX.

XXIII. Lattice polyhedra (Hoffman [1976,1978], Hoffman and Schwartz [1978]): => I, II,IV,XIII,XVI.

XXIV. A more general framework (Schrijver [1982]): $=>$ XIX, XX, XXIII.

XXV.Grishuhin's model (Grishuhin [1981]): $=>$ XX.

I. KONIG'S MATCHING AND COVERING THEOREMS (Konig [1931,1932]). Let $G=(V, E)$ be a bipartite graph. Then the maximum size of a matching in $G$ is equal to the minimum number of points needed to cover all edges. Moreover, the maximum size of a stable set of points in $G$ is equal to the minimum number of edges in $G$ needed to cover all points (assuming $V=U E$ ).

More generally, for all $d_{1}, d_{2}: V \rightarrow R$, the system

$$
d_{1}(v) \leq x(\delta(v)) \leq d_{2}(v) \quad(v \in V)
$$

is t.d.i., even box-t.d.i. This contains weighted extensions of the Konig-theorems, like optimal assignment and transportatin problems, and can be derived easily from the total unimodularity of the constraint matrix.

II. MENGER'S THEOREM AND FLOW AND CIRCULATION PROBLEMS. Let $D=(V, A)$ be a directed graph, and let $r$ and $s$ be two points of $D$. Then the maximum number of pairwise arc-disjoint $r-s-$ paths is equal to the minimum size of an $r-s$-cut (Menger [1927]). A weighted version is the max-flow min-cut theorem (Ford and Fulkerson [1956], Elias, Feinstein and Shannon [1956]).

More generally, the system

$$
x\left(8^{+}(v)\right)=x\left(8^{-}(v)\right) \quad(v \in V)
$$

is box-t.d.i. This contains min-cost flow and circulation problems (cf. Hoffman [1960]), and Dilworth's theorem and its extensions of Greene and Kleitman (see IV below).

Even more generally, for all $d_{1}, d_{2}: V \rightarrow R$, the system

$$
d_{1}(v) \leq x\left(8^{+}(v)\right)-x\left(8^{-}(v)\right) \leq d_{2}(v) \quad(v \in V)
$$

is box-t.d.i., which follows directly from the total unimodularity of the constraint matrix. Clearly, (1) is a special case of (3). In fact, a direct construction gives the converse implication (Hoffman [1960], Ford and Fulkerson [1958]).

Indeed, to derive the box-total dual integrality of (3) from that of 
(1), replace each point $v$ by two new points $v^{\prime}$ and $v^{\prime \prime}$, each $\operatorname{arc}(v, w)$ by the new arc $\left(v^{\prime}, w^{\prime \prime}\right)$, and add arcs $\left(v^{\prime}, v^{\prime \prime}\right)$ for all $v$ in $v$. This makes the directed graph $\left(V^{*}, A^{*}\right)$. Then the box-t.d.i.ity of $(3)$ is equivalent to that of

$$
\begin{aligned}
& x\left(8_{A^{-}}^{-}\left(v^{\prime \prime}\right)\right)=0 \quad(v \in V), \\
& d_{1}(v) \leq x\left(\delta_{A^{+}}^{+}\left(v^{\prime}\right)\right) \leq d_{2}(v) \quad(v \in V),
\end{aligned}
$$

which follows from I.

III. SHORTEST PATHS. Let $D=(V, A)$ be a digraph, and let $r$ and $s$ be points of $D$. Then the minimum length of an $r-s-$ path is equal to the maximum number of pairwise disjoint $r-s$-cuts. This follows easily, e.g. , from Dijkstra's shortest path algorithm. Its weighted version (sometimes called the max-potential min-work theorem) is equivalent to the total dual integrality of the following system:

$$
\begin{aligned}
& x(a) \geq 0 \quad(a \in A), \\
& x\left(8^{-}\left(V^{\prime}\right)\right) \geq 1 \quad\left(s \in V^{\prime} \subseteq n\{r\}\right),
\end{aligned}
$$

which also may be seen as special case of (2).

IV. PARTITIONS OF PARTIALLY ORDERED SETS. Let $(P, \leq)$ be a partially ordered set. Then the maximum size of an antichain is equal to the minimum number of chains needed to cover $\boldsymbol{P}$ (Dilworth [1950]). This is equivalent (by splitting elements of $P$ ) to the total dual integrality of the following system:

$$
\begin{array}{ll}
x(p) \geq 0 & (p \in P), \\
x(C) \leq 1 & (C \subseteq P, C \text { chain }) .
\end{array}
$$

Dilworth's theorem may be derived from the box-total integrality of (2) (Fulkerson [1956]).

Indeed, let for each $p$ in $P, p^{\prime}$ and $p^{\prime \prime}$ be two points, and let there be arcs $\left(p^{\prime}, p^{\prime \prime}\right)$ for all $p$ in $P$, and $\left(p^{\prime \prime}, q^{\prime}\right)$ for all $p<q$ in $P$. Moreover, let there be two new points $r$ and $s$, and ares $(s, r),\left(r, p^{\prime}\right)$ and $\left(p^{\prime \prime}, s\right)$ for all $p$ in $P$. This makes the digraph $D=(V, A)$. Then II gives the total dual integrality of the system:

$$
\begin{array}{ll}
x(a) \geq 0 & (a \in A), \\
x\left(\delta^{-}(v)\right)=x\left(\delta^{+}(v)\right) & (v \in V), \\
x\left(\left(p^{\prime}, p^{\prime \prime}\right)\right) \geq 1 & (p \in P) .
\end{array}
$$

Minimizing $x((s, r))$ over (7) gives Dilworth's theorem.

An easier "polar" theorem is: the maximum size of a chain in a 
partially ordered set $(P, S)$ is equal to the minimum number of antichains needed to cover $P$. Equivalently, the following system is totally dual integral:

$$
\begin{array}{ll}
x(p) \geq 0 & (p \in P), \\
x(A) \leq 1 & (A \subseteq P, \text { A antichain). }
\end{array}
$$

Greene and Kleitman [1976] showed more generally that, for any natural number $k$, the maximum size of the union of $k$ antichains in a partially ordered set $(P, S)$ is equal to

$$
\text { min's } P\left|P P^{\prime}\right|+k . \gamma\left(P^{\prime}\right) \text {, }
$$

where $\gamma\left(P^{\prime}\right)$ denotes the minimum number of chains needed to cover $P^{\prime}$. This is equivalent to the total dual integrality of the system:

$$
\begin{array}{lc}
0 \leq x(p) \leq 1 & (p \in P), \\
x(C) \leq k & (\mathrm{C} \subseteq P, \mathrm{C} \text { chain }) .
\end{array}
$$

(To see the equivalence, the easy theorem mentioned above may be used.) (10) of course generalizes (6), and again the total dual integrality of (10) can be derived from that of (2) (min-cost circulation; cf. Frank [1980a]).

Indeed, add to the graph $D$ described above (after Dilworth's theorem), new $\operatorname{arcs}\left(p^{\prime \prime}, p^{\prime}\right)$ for all $p$ in $P$, thus yielding the digraph $D^{*}=\left(V, A^{*}\right)$. Then II gives the total dual integrality of the system

$$
\begin{aligned}
& x(a) \geq 0 \quad\left(a \in A^{*}\right), \\
& x\left(\left(p^{\prime}, p^{\prime \prime}\right)\right) \geq 1 \quad(p \in P), \\
& x\left(\delta_{A^{*}}^{-}(v)\right)=x\left(\delta_{A^{*}}^{+}(v)\right) \quad(v \in V) .
\end{aligned}
$$

Minimizing $k \cdot x((s, r))+\sum_{p \in P} x\left(\left(p^{\prime \prime}, p^{\prime}\right)\right)$ over (11) gives Greene and Kleitman's result.

Greene [1976] showed that again "chain" and "antichain" may be interchanged: for any natural number $k$, the maximum size of the union of $k$ chains in a partially ordered set $(P, S)$ is equal to

$$
\min _{P^{\prime} \subseteq}\left|P \backslash P^{\prime}\right|+k . \alpha\left(P^{\prime}\right),
$$

where $\alpha\left(P^{\prime}\right)$ denotes the minimum number of antichains needed to cover $P^{\prime}$. Using Dilworth's theorem, this is equivalent to the total dual integrality of the system:

$$
0 \leq x(p) \leq 1 \quad(p \in P),
$$




$$
x(A) \leq k \quad(A \subseteq P, A \text { antichain })
$$

Again this can be derived from the box total dual integrality of (2) (min-cost circulation; cf. Frank [1980a]).

Indeed, let $D$ be as above. Then Greene's result follows from the total dual integrality of:

$$
\begin{array}{ll}
x(a) \geq 0 \quad(a \in A), & \\
x\left(8_{A}^{+}(v)\right)=x\left(8_{A}^{-}(v)\right) & (v \in V), \\
x\left(\left(p^{\prime}, p^{\prime \prime}\right)\right) \leq 1 & (p \in P), \\
x((s, r)) \leq k . &
\end{array}
$$

This can be seen by maximizing $\Sigma_{p} x\left(\left(p^{\prime}, p^{\prime \prime}\right)\right)$ over (14).

One similarly shows that the systems

$$
x(\mathrm{C}) \leq k \quad(\mathrm{C} \subseteq P, \mathrm{C} \text { chain })
$$

and

$$
x(A) \leq k \quad(A \subseteq P, A \text { antichain })
$$

are box totally dual integral.

For a generalization, see LIII (the Hoffman-Schwartz chain model) in Section 3.

Konig's covering theorem (I) is a corollary of Dilworth's theorem. In turn, Dilworth's theorem may be derived from Konig's theorem in a way similar to that given in II (cf. Ford and Fulkerson [1962] and Mirsky and Perfect [1966]).

V. COMMONS SDR'S. Two families of subsets $A_{1}, \ldots, A_{n}$ and $B_{1}, \ldots, B_{n}$ of a finite set $S$ have a common SDR, if and only if

$$
\left|\bigcup_{i \in J} A_{i} \cap \bigcup_{j \in J} B_{j}\right| \geq|I|+|J|-n
$$

for all $I, J \subseteq\{1, \ldots, n\}$ (Ford and Fulkerson [1958]). More generally, the following system is box totally dual integral.

$$
\begin{array}{ll}
x\left(S^{\prime}\right) \leq \text { number of } i \text { with } A_{i} \cap S^{\prime} \neq \varnothing \quad\left(S^{\prime} \subseteq S\right), \\
x\left(S^{\prime}\right) \leq \text { number of } j \text { with } B_{j} \cap S^{\prime} \neq \varnothing \quad\left(S^{\prime} \subseteq S\right) .
\end{array}
$$

This may be derived from the box total dual integrality of (2) by defining a suitable graph.

Note that Konig's matching theorem (I) is a special case.

VI. TREES AND FORESTS IN UNDIRECTED GRAPHS. Let 
$G=(V, E)$ be an undirected graph, and let $l: E \rightarrow \boldsymbol{Z}_{+}$be a length function. Then the maximum length of a subforest in $G$ is equal to the minimum value of $\sum_{i=1}^{i}\left(\left|V_{i}\right|-1\right)$, where $V_{1}, \ldots, V_{i}$ are nonempty subsets of $V(t \geq 0)$ such that each edge $e$ of $G$ is contained in at least $l(e)$ of the $V_{l}$. This may be derived easily from the greedy algorithm - see Edmonds [1970]. Similarly, one derives more generally that the following system is box totally dual integral:

$$
\left.x\left(<V^{\prime}\right\rangle\right) \leq\left|V^{\prime}\right|-1 \quad\left(\varnothing \neq V^{\prime} \subseteq V\right),
$$

where $\left\langle V^{\prime}\right\rangle$ denotes the set of edges contained in $V^{\prime}$.

Also from the greedy method one may derive: if $G$ is connected, the minimum length of a spanning tree is equal to the maximum value of $\sum_{i=1}^{t}\left(\left|\Pi_{l}\right|-1\right)$, where $\Pi_{1}, \ldots, \Pi_{t}$ are partitions of $V$ into nonempty classes $(t \geq 0)$ such that an edge $e$ connects distinct classes of at most $l(e)$ of the $\Pi_{i}$. More generally, the system

$$
x(\epsilon(\Pi)) \geq|\Pi|-1 \quad \text { (II partition of } V \text { into nonempty classes)(18) }
$$

is box totally dual integral, where $\epsilon$ (II) denotes the set of edges connecting distinct classes of $\Pi$. Again this follows with the greedy method, or alternatively, from the box dual integrality of (17).

VII. OPTIMUM ARBORESCENCES. Let $D=(V, A)$ be a directed graph, let $r$ be a point of $D$, and let $l: A \rightarrow Z_{+}$be a length function. Then the minimum length of an $r$-arborescence is equal to the maximum number $t$ of $r$-cuts $C_{1}, \ldots, C_{t}$ such that no arrow $a$ is in more than $l(a)$ of these $r$-cuts (Fulkerson [1974], cf. Edmonds [1967]). [An $r$-arborescence is a rooted spanning tree with root $r$. An $r$-cut is a set of the form $\delta^{-}\left(V^{\prime}\right)$, with $\left.\varnothing \neq V^{\prime} \subseteq U\{r\}.\right]$

Equivalently, the following system is totally dual integral:

$$
\begin{array}{lr}
x(a) \geq 0 \quad(a \in A), \\
x\left(8^{-}\left(V^{\prime}\right)\right) \geq 1 & \left(\varnothing \neq V^{\prime} \subseteq V\{r\}\right) .
\end{array}
$$

One may derive variations, e.g., on the maximum length of an $r$ arborescence, on the minimum length of an arborescence (where the root is not specified in advance), and on the maximum length of a branching (which is a set of arcs each component of which forms a rooted tree).

The results on shortest paths (III) and spanning (undirected) trees (VI) are special cases of Fulkerson's theorem and of the total dual integrality of (19).

Frank [1979a] showed more generally that the system 


$$
\begin{aligned}
& x(a) \geq 0 \quad(a \in A), \\
& x\left(\delta^{-}\left(V^{\prime}\right)\right) \geq k \quad\left(\varnothing \neq V^{\prime} \subseteq n\{r\}\right)
\end{aligned}
$$

is box totally dual integral, for each number $k$.

VIII. OPTIMUM BI-BRANCHINGS. Let $D=(V, A)$ be a directed graph, let $V$ be partitioned into classes $U$ and $W$, and let $l: A \rightarrow \boldsymbol{Z}_{+}$be a length function. Then the minimum length of a bi-branching is equal to the maximum number $t$ of $(U, W)$-cuts $C_{1}, \ldots, \mathbf{C}_{t}$ such that each arc $a$ is in at most $l(a)$ of these cuts (Schrijver [1982a]). [Here a bibranching (with respect to $U, W$ ) is a set $A^{\prime}$ of arcs such that each point in $U$ is the end point of a directed path in $A^{\prime}$ starting in $W$, and each point of $W$ is the starting point of a directed path in $A^{\prime}$ ending in $U$. A $(U, W)$-cut is a set of the form $\delta^{-}\left(V^{\prime}\right)$ with $\varnothing \neq V^{\prime} \subseteq U$ or $U \subseteq V^{\prime} \neq V$.] Equivalently, the following system is totally dual integral:

$$
\begin{array}{ll}
x(a) \geq 0 & (a \in A), \\
x\left(\delta^{-}\left(V^{\prime}\right)\right) \geq 1 \quad\left(\varnothing \neq V^{\prime} \subseteq U \text { or } U \subseteq V^{\prime} \neq V\right) .
\end{array}
$$

This contains Konig's edge covering theorem (I), shortest paths (III), spanning undirected trees (VI), optimum branchings (VII).

More generally, the system:

$$
\begin{array}{ll}
x(a) \geq 0 & (a \in A), \\
x\left(8^{-}\left(V^{\prime}\right)\right) \geq k & \left(\varnothing \neq V^{\prime} \subseteq U \text { or } U \subseteq V^{\prime} \neq V\right)
\end{array}
$$

is box totally dual integral, for each number $k$. Clearly, (22) contains (20).

IX. LUCCHESI-YOUNGER THEOREM. Let $D=(V, A)$ be a directed graph. Then the minimum number of arcs needed to "cover" (i.e., intersect) all directed cuts is equal to the maximum number of pairwise directed cuts (Lucchesi and Younger [1978], cf. Lovász [1976]). [Here a directed cut is a set of edges of the form $\delta^{-}\left(V^{\prime}\right)$, with $\varnothing \neq V^{\prime} \neq V$ and $\delta^{+}\left(V^{\prime}\right)=\varnothing$.] By replacing ares by ares in series one derives a weighted version, which is equivalent to the total dual integrality of the system:

$$
\begin{aligned}
& x(a) \geq 0 \quad(a \in A), \\
& x\left(\delta^{-}\left(V^{\prime}\right)\right) \geq 1 \quad\left(\varnothing \neq V^{\prime} \neq V, \delta^{+}\left(V^{\prime}\right)=\varnothing\right) .
\end{aligned}
$$

By taking $D$ to be a complete directed bipartite graph Konig's covering theorem (I) directly follows. More corollaries follow through the theorem of optimum strong connectors derived in $X$ from the 
Lucchesi-Younger theorem.

Edmonds and Giles [1977] showed more generally the box total dual integrality of the systems

$$
x\left(8^{-}\left(V^{\prime}\right)\right) \geq k \quad\left(\varnothing \neq V^{\prime} \neq V, 8^{+}\left(V^{\prime}\right)=\varnothing\right),
$$

and

$$
x\left(8^{-}\left(V^{\prime}\right)\right) \leq k \quad\left(\varnothing \neq V^{\prime} \neq V, \delta^{+}\left(V^{\prime}\right)=\varnothing\right),
$$

for each number $k$. A special case is, e.g., Greene's result on unions of chains (IV), which is equivalent to (25) if we add $0 \leq x \leq 1$.

X. OPTIMUM STRONG CONNECTORS. Let $D=(V, A)$ and $D_{0}=\left(V, A_{0}\right)$ be directed graphs, such that for each $\operatorname{arc}(v, w)$ of $D$ a there exist $v^{\prime}$ and $w^{\prime}$ in $V$ and directed paths in $D_{0}$ from $v$ to $v^{\prime}$, from $w^{\prime}$ to $v^{\prime}$, and from $w^{\prime}$ to $w$ (the points $v, v^{\prime}, w^{\prime}, w$ need not be distinct). Let $l: A \rightarrow \boldsymbol{Z}_{+}$be a length function. Then the minimum length of a strong connector is equal to the maximum number $t$ of nonempty proper subsets $V_{1}, \ldots, V_{t}$ of $V$, such that no arc of $D_{0}$ enters any of the $V_{i}$, and such that each arc $a$ of $D$ enters at most $l(a)$ of the $V_{i}$ (Schrijver [1982a]). [Here a strong connector (for $D_{0}$ ) is a set $A^{\prime}$ of arcs of $D$ such that the digraph $\left(V, A_{0} \cup A^{\prime}\right)$ is strongly connected.]

Equivalently, the following system is totally dual integral:

$$
\begin{aligned}
& x(a) \geq 0 \quad(A \in A), \\
& x\left(\delta_{A}^{-}\left(V^{\prime}\right)\right) \geq 1 \quad\left(\varnothing \neq V^{\prime} \neq V, \delta_{\Lambda_{0}}^{-}\left(V^{\prime}\right)=\varnothing\right) .
\end{aligned}
$$

In fact this may be derived from the Lucchesi-Younger theorem (IX).

Indeed, extend $D_{0}$ as follows. For each arc $a=(v, w)$, choose points $v^{\prime}$ and $w^{\prime}$ in $V$ satisfying the above property. Let $v_{a}$ and $w_{a}$ be two new points, and add arcs $\left(v, v_{a}\right),\left(w_{a}, v_{a}\right),\left(w_{a}, w\right),\left(v_{a}, v^{\prime}\right)$, $\left(w^{\prime}, w_{c}\right)$. This makes the digraph $D_{1}$. Let the length of $\left(w_{a}, v_{c}\right)$ be $l(a)$, and let all other arcs of $D_{1}$ have very large length. Then the minimum length of a covering of the directed cuts in $D_{1}$ is equal to the minimum length of a strong connector in $D$.

In turn, the strong connector result contains the Lucchesi-Younger theorem (IX), and also Konig's covering theorem (I), shortest paths (III), optimum branchings (VII), and optimum bi-branchings (VIII).

More generally, the following system is box totally dual integral, for each number $k$ :

$$
\begin{array}{ll}
x(a) \geq 0 & (a \in A), \\
x\left(\delta_{A}^{-}\left(V^{\prime}\right)\right) \geq k & \left(\varnothing \neq V^{\prime} \neq V, \delta_{A_{0}}^{-}\left(V^{\prime}\right)=\varnothing\right) .
\end{array}
$$


Clearly, (27) contains (20) and (22).

XI. ORIENTATIONS OF UNDIRECTED GRAPHS. Each $2 k$ connected undirected graph has a $k$-connected orientation (NashWilliams [1969]). Frank [1980] observed the relation of NashWilliams' theorem with that of Edmonds and Giles [1977] (cf. XX), yielding the following more general result: given a directed graph $D=(V, A)$ and a natural number $k$, then the following system is totally dual integral:

$$
\begin{aligned}
& 0 \leq x(a) \leq 1 \quad(a \in A), \\
& x\left(8^{+}\left(V^{\prime}\right)\right)-x\left(8^{-}\left(V^{\prime}\right)\right) \geq k-\left|8^{-}\left(V^{\prime}\right)\right|
\end{aligned}
$$

The fact that this indeed generalizes Nash-Williams' result can be seen as follows (cf. Frank and Tardos [1981]). Direct the edges of the $2 k$-connected undirected graph in an arbitrary way, yielding the digraph $D=(V, A)$. Now (28) has a solution as $x=\frac{1}{2}$ satisfies (28). Hence there exists an integral vector $x$ satisfying (28). Let $D^{\prime}$ arise from $D$ by reversing the orientation of the arcs $a$ of $D$ with $x(a)=1$. Then $D^{\prime}$ is $k$-connected.

In fact, (28) yields a min-max result for "min-cost orientations".

XII. MATROIDS, POLYMATROIDS AND SUBMODULAR FUNCTIONS. Let $M=(S, I)$ be a matroid, with rank function $p$, and let $w: S \rightarrow z_{+}$be a weight function. Then the maximum weight of an independent set is equal to the minimum value of $\sum_{i=1}^{i} \rho\left(S_{i}\right)$, where $S_{1}, \ldots, S_{t}$ are subsets of $S$ such that each element $s$ of $S$ is contained in at least $w(s)$ of the $S_{i}$. This follows easily from the greedy algorithm see Edmonds [1970,1971]. It contains the results on trees (VI) as special cases.

More generally, for any submodular function $f: P(S) \rightarrow R_{+}$, the following system is totally dual integral (Edmonds [1970]):

$$
\begin{array}{rlrl}
x(s) & \geq 0 & & (s \in S), \\
x\left(S^{\prime}\right) \leq f\left(S^{\prime}\right) & & \left(S^{\prime} \subseteq S\right) .
\end{array}
$$

Again, this can be derived with the greedy method. The solution set of (29) is called the polymatroid (polytope) corresponding to $f$. The set of all solutions $x$ of $(29)$ which satisfy $x(S)=f(S)$ is nonempty, and is called the principal face. If $F$ is the principal face of the polymatroid $P$, then $P=\{x \geq 0 \mid x \leq y$ for someyin $F\}$.

Even more generally, let $C$ be an intersecting family on $S$ and let $f: \mathbf{C} \rightarrow \boldsymbol{R}$ be submodular on intersecting pairs. Then the system 


$$
x\left(S^{\prime}\right) \leq f\left(S^{\prime}\right) \quad\left(S^{\prime} \in \mathbf{C}\right)
$$

is box totally dual integral. This may be derived from the total dual integrality of (29).

Indeed, let $c_{1}, c_{2}: S \rightarrow R$. To prove the total dual integrality of

$$
\begin{array}{lr}
c_{1}(s) \leq x(s) \leq c_{2}(s) & (s \in S), \\
x\left(S^{\prime}\right) \leq f\left(S^{\prime}\right) & \left(S^{\prime} \in C\right),
\end{array}
$$

we may assume that $c_{1}=0$, as total dual integrality is invariant under translations and submodularity under the addition of modular functions. Moreover, we may assume that (31) has a solution, and that $\{s\} \in C$ and $f(\{s\}) \leq c_{2}(s)$ for all $s$ in $S$ (as adding $\{s\}$ to $C$ and replacing/defining $f(\{s\})$ by $\min \left\{f(\{s\}), c_{2}(s)\right\}$ keeps $C$ and $f$ to have the required properties and does not change the system (31). Now define $f^{\prime}: P(S) \rightarrow R$ by

$$
f^{\prime}\left(S^{\prime}\right):=\min \sum_{i=1}^{\prime} f\left(S_{l}\right),
$$

for $S^{\prime} \subseteq S$, where the minimum ranges over all collections of pairwise disjoint sets $S_{1}, \ldots, S_{t}$ in $C$ with $S^{\prime} \subseteq S_{1} \cup \cdots \cup S_{t}$. Then the total dual integrality of (31) is equivalent to the total dual integrality of (29) for $f$ " instead of $f$ (note that the "new" constraints are nonnegative integer combinations of the "old" constraints).

One similarly derives that if $f \geq 0$ and $\cup \mathrm{C}=S$, and if we add the condition $x \geq 0$ to (30), the solution set will be exactly the polymatroid corresponding to the function $f^{\prime}$ as defined in (32) (cf. Dunstan [1976], Lovász [1977]). A solution set of a system of form (30) is called an extended polymatroid. system

Note that the box total dual integrality of (30) implies that of the

$$
\begin{aligned}
x\left(S^{\prime}\right) & \leq f\left(S^{\prime}\right) \quad\left(S^{\prime} \in C\right), \\
x(S) & =k
\end{aligned}
$$

for any number $k$.

XIII. (POLY)MATROID INTERSECTION. Let $M_{1}=\left(S, I_{1}\right)$ and $M_{2}=\left(S, I_{2}\right)$ be matroids, with rank functions $p_{1}$ and $p_{2}$, respectively. Then the maximum size of a common independent set is equal to the minimum value of $p_{1}\left(S^{\prime}\right)+p_{2}\left(S S^{\prime}\right)$, for $S^{\prime} \subseteq S$ (Edmonds $[1970,1979])$. A weighted version gives the total dual integrality of the following system:

$$
x(s) \geq 0 \quad(s \in S),
$$




$$
\begin{array}{ll}
x\left(S^{\prime}\right) \leq \rho_{1}\left(S^{\prime}\right) & \left(S^{\prime} \subseteq S\right), \\
x\left(S^{\prime}\right) \leq p_{2}\left(S^{\prime}\right) & \left(S^{\prime} \subseteq S\right) .
\end{array}
$$

More generally, if $f_{1}$ and $f_{2}$ are submodular set-functions on $S$, the system

$$
\begin{array}{ll}
x(s) \geq 0 & (s \in S), \\
x\left(S^{\prime}\right) \leq f_{1}\left(S^{\prime}\right) & \left(S^{\prime} \subseteq S\right), \\
x\left(S^{\prime}\right) \leq f_{2}\left(S^{\prime}\right) & \left(S^{\prime} \subseteq S\right)
\end{array}
$$

is totally dual integral (which may be derived from that of (34) by splitting elements of $S$ ).

Even more generally, if $C_{1}$ and $C_{2}$ are intersecting families on $S$, and if $f_{1}: \mathbf{C}_{1} \rightarrow \boldsymbol{R}$ and $f_{2}: \mathbf{C}_{2} \rightarrow \boldsymbol{R}$ are submodular on intersecting pairs, the following system is box totally dual integral:

$$
\begin{array}{ll}
x\left(S^{\prime}\right) \leq f_{1}\left(S^{\prime}\right) & \left(S^{\prime} \in C_{1}\right), \\
x\left(S^{\prime}\right) \leq f_{2}\left(S^{\prime}\right) & \left(S^{\prime} \in C_{2}\right) .
\end{array}
$$

This may be derived from the total dual integrality of (35) in the same way as in XII.

Again, similar results hold if we replace "submodular" by "supermodular". Moreover, if $C_{1}$ and $C_{2}$ are interesecting families on $S$, and $f: C_{1} \rightarrow R$ is submodular on intersecting pairs and $g: C_{2} \rightarrow R$ is supermodular on intersecting pairs, then the system

$$
\begin{array}{ll}
x\left(S^{\prime}\right) \leq f\left(S^{\prime}\right) & \left(S^{\prime} \in C_{1}\right), \\
x\left(S^{\prime}\right) \geq g\left(S^{\prime}\right) & \left(S^{\prime} \in C_{2}\right)
\end{array}
$$

is box totally dual integral. Another nice application (in fact, equivalent form) of the polymatroid intersection theorem is Frank's "Discrete separation theorem": if $f$ is a submodular set-function on $S$, and $g$ is a supermodular set-function on $S$, such that $f \geq 8$, then there is a modular set-function $h$ on $S$ with $f \geq h \geq g$.

This scheme contains as special cases: matchings and coverings in bipartite graphs (I), common SDRs (V), optimum arborescences (VII) (also optimum branchings), and of course those mentioned in XII. Through the constructions given below all other results from I-XII will follow as special cases.

XIV. SUBMODULAR FUNCTIONS ON CROSSING FAMIIIES. Let $\mathbf{C}$ be a crossing family, let $f: \mathbf{C} \rightarrow \boldsymbol{R}$ be submodular on crossing pairs, and let $k$ be a number. Then the following system is totally dual 
integral (Frank and Tardos [1981]):

$$
\begin{aligned}
& x\left(S^{\prime}\right) \leq f\left(S^{\prime}\right) \quad\left(S^{\prime} \in C\right), \\
& x(S)=k .
\end{aligned}
$$

The total dual integrality of (38) follows from that of (30) (cf. Frank and Tardos [1981], Frank [1982]).

Indeed, let $C^{\prime}$ be the collection of all subsets of $S$ of the form $S_{1} \cap \cdots \cap S_{t}$, where $S_{1}, \ldots, S_{t}$ are sets in $C$ such that $S_{l} \cup S_{j}=S$ for all $1 \leq i<j \leq t$. Define for $S^{\prime}$ in $C^{\prime}$,

$$
f^{\prime}\left(S^{\prime}\right)=k-\max \sum_{i=1}^{k}\left(k-f\left(S_{i}\right)\right),
$$

where the maximum ranges over all collections $S_{1}, \ldots, S_{t}$ as before with $S^{\prime}=S_{1} \cap \cdots \cap S_{t}$. Then $C^{\prime}$ is an intersecting family, and $f^{\prime}$ is submodular on intersecting pairs. Now the total dual integrality of (38) follows from that of the equivalent system

$$
\begin{aligned}
& x\left(S^{\prime}\right) \leq f^{\prime}\left(S^{\prime}\right) \quad\left(S^{\prime} \in C^{\prime}\right), \\
& x(S)=k,
\end{aligned}
$$

which follows from (33).

Conversely, the model XII can be derived by extension of the set $S$ by one new element (taking $k=0$ ).

One similarly shows that if (38) is solvable and contained in the nonnegative orthant, it is the principal face of a polymatroid.

Note that the systems of form (38) are closed under "intersection with boxes", i.e., if we add constraints like $c_{1} \leq x \leq c_{2}$, the new system can be described in the form (38).

Indeed, extension of $\mathbf{C}$ with singletons or complements of singletons keeps $\mathrm{C}$ to be a crossing family. Moreover, decreasing $f$ on singletons and increasing $f$ on complements of singletons, keeps $f$ to be submodular on crossing pairs.

Moreover, if $\boldsymbol{g}: \mathbf{C} \rightarrow \boldsymbol{R}$ is supermodular on crossing pairs, the system " $x\left(S^{\prime}\right) \geq g\left(S^{\prime}\right)$ (S'EC), $x(S)=k$ " is equivalent to a system of form (38) (by defining $f\left(S^{\prime}\right)=k-g\left(S S^{\prime}\right)$ ).

In the same way as above one derives from the total dual integrality of (36) that intersections of two systems of the form (38) are totally dual integral. That is, if $\mathbf{C}_{1}$ and $\mathbf{C}_{2}$ are crossing families on $S$, and $f_{1}: \mathbf{C}_{1} \rightarrow \boldsymbol{R}$ and $f_{2}: \mathbf{C}_{2} \rightarrow \boldsymbol{R}$ are submodular on crossing pairs, and $k$ is a number, then the system

$$
x\left(S^{\prime} \leq f_{1}\left(S^{\prime}\right) \quad\left(S^{\prime} \in C_{1}\right),\right.
$$




$$
\begin{array}{ll}
x\left(S^{\prime}\right) \leq f_{2}\left(S^{\prime}\right) & \left(S^{\prime} \in C_{2}\right), \\
x(S)=k &
\end{array}
$$

is box totally dual integral.

Conversely, the box total dual integrality of (36) follows from that of (41) by extension of $S$ by one new element, and taking $k=0$.

This result will turn out to be a crucial link in this list: at one side it may be derived by a direct construction from polymatroid intersec. tion, at the other side many of the other results can be reduced to it.

Thus it contains the Lucchesi-Younger theorem (IX) (and hence by the construction given in X also optimum strong connectors) - see Frank and Tardos [1981].

Indeed, let $D=(V, A)$ be a directed graph. Add for each arc $a=(v, w)$ of $D$ a new arc $a^{\prime}=(w, v)$. This makes the digraph $D^{\prime}=\left(V, A \cup A^{\prime}\right)$. Let $C_{1}=\left\{\left\{a, a^{\prime}\right\} \mid a \in A\right\}$ and $g_{1}\left(\left\{a, a^{\prime}\right\}\right)=1$ for $a$ in $A$. For each subset $V^{\prime}$ of $V$, let $H\left(V^{\prime}\right)$ denote the set of ares of $D^{\prime}$ with head in $V^{\prime}$, let $C_{2}=\left\{H\left(V^{\prime}\right) \mid \varnothing \neq V^{\prime} \neq V, \delta_{A}^{+}\left(V^{\prime}\right)=\varnothing\right\}$, and let $g_{2}\left(H\left(V^{\prime}\right)\right)=1+$ the number of ares of $D$ contained in $V^{\prime}$. Then from (41), the system

$$
\begin{aligned}
& x\left(\left\{a, a^{\prime}\right\}\right) \geq g_{1}\left(\left\{a, a^{\prime}\right\}\right) \quad(a \in A), \\
& x\left(H\left(V^{\prime}\right)\right) \geq g_{2}\left(H\left(V^{\prime}\right)\right) \\
& x\left(A \cup A^{\prime}\right)=|A|
\end{aligned}
$$

is box totally dual integral. This system is TDI-equivalent to:

$$
\begin{array}{ll}
x\left(\left\{a, a^{\prime}\right\}\right)=1 & (a \in A), \\
x\left(8_{A}^{-}\left(V^{\prime}\right)\right) \geq 1 & \left(\varnothing \neq V^{\prime} \neq V, \delta_{A}^{+}\left(V^{\prime}\right)=\varnothing\right) .
\end{array}
$$

This implies that the system (23) is totally dual integral.

One similarly shows that the system (28) is totally dual integral, and that hence Nash-Williams' theorem follows. Clearly, also polymatroid intersection (XII), and the special cases mentioned there, are contained in this model.

XV. GENERALIZED POLYMATROIDS (Frank [1981b]). Let B and $P$ be intersecting families on a set $S$, and let $b: B \rightarrow R$ and $p: \mathbb{P} \rightarrow \boldsymbol{R}$ be functions such that:

(i)b is submodular on intersecting pairs,

(ii)p is supermodular on intersecting pairs,

(iii) if $S^{\prime} \in B, S^{\prime \prime} \in P$ and $S^{\prime} S^{\prime \prime} \neq \varnothing, S^{\prime \prime} S^{\prime} \neq \varnothing$, then $S^{\prime} S^{\prime \prime} \in B$, 
$S^{\prime \prime} S^{\prime} \in \mathbf{P}$, and $b\left(S^{\prime}\right)-p\left(S^{\prime \prime}\right) \geq b\left(S^{\prime} W^{\prime \prime}\right)-p\left(S^{\prime \prime} \backslash S^{\prime}\right)$.

Then the following system is box totally dual integral:

$$
\begin{array}{ll}
x\left(S^{\prime}\right) \leq b\left(S^{\prime}\right) & \left(S^{\prime} \in \mathbf{B}\right), \\
x\left(S^{\prime}\right) \geq p\left(S^{\prime}\right) & \left(S^{\prime} \in \mathbf{P}\right) .
\end{array}
$$

This may be derived from the total dual integrality of (38).

To this end, extend $S$ by one new element $r$. Let $\mathbf{C}=\mathbf{B} \cup\left\{r \cup\left(S S^{\prime}\right) \mid S^{\prime} \in \mathbf{P}\right\}$, let $f\left(S^{\prime}\right)=b\left(S^{\prime}\right)$ for $S^{\prime}$ in $B$, and $f\left(\{r\} \cup\left(S S^{\prime}\right)\right)=-p\left(S^{\prime}\right)$ for $S^{\prime}$ in $\mathbf{P}$. Then $\mathbf{C}$ is a crossing family, and $f$ is submodular on crossing pairs. Moreover, the box total dual integrality of (45) is equivalent to the box total dual integrality of (38).

Similarly the converse implication can be derived.

The solution set of (45) is called a generalized polymatroid. The above construction shows that generalized polymatroids are exactly the projections on a basis hyperplane of the principal face of an extended polymatroid. It may be seen easily that the inequality systems (29), (30) (together with their supermodular variants), (33), (38) form special cases of (45) (so generalized polymatroids generalize polymatroids). Moreover, systems of the form (45) are closed again under adding box inequalities (like $c_{1} \leq x \leq c_{2}$ ).

Even more generally, also if we intersect systems of form (45) we retain total dual integrality. That is, if we have two pairs of collections $B_{1}, P_{1}$ and $B_{2}, P_{2}$ and two pairs of functions $b_{1}, p_{1}$ and $b_{2}, p_{2}$, each pair satisfying (44), then the following system is box totally dual integral:

$$
\begin{array}{ll}
x\left(S^{\prime}\right) \leq b_{1}\left(S^{\prime}\right) & \left(S^{\prime} \in B_{1}\right), \\
x\left(S^{\prime}\right) \geq p_{1}\left(S^{\prime}\right) & \left(S^{\prime} \in \mathbb{P}_{1}\right), \\
x\left(S^{\prime}\right) \leq b_{2}\left(S^{\prime}\right) & \left(S^{\prime} \in B_{2}\right), \\
x\left(S^{\prime}\right) \geq p_{2}\left(S^{\prime}\right) & \left(S^{\prime} \in \mathbb{P}_{2}\right) .
\end{array}
$$

This can be derived, in the same way as above, from the total dual integrality of (41).

(46) contains as special cases (1) (matchings and coverings in bipartite graphs (I)), (16), (common SDR's (V)), (17), (18) (trees and forests (VI)), (29), (30), (33) ((extended) polymatroids (XI)), (34), (35), (36), (37) (polymatroid intersection (XIII)), (38), (41) (submodular functions on crossing families), and (45).

XVI. BLOCKERS OF COMMON INDEPENDENT SETS. Groflin 
and Hoffman [1981] showed the following. Let $f_{1}$ and $f_{2}$ be submodular set functions on a set $S$. Define

$$
f\left(S^{\prime}\right)=\min _{\gamma^{\prime \prime} \subseteq S^{\prime}} f_{1}\left(S^{\prime \prime}\right)+f_{2}\left(S^{\prime} \backslash S^{\prime \prime}\right),
$$

for $S^{\prime} \subseteq S$. Then for any number $k$, the system

$$
x\left(S^{\prime}\right) \geq k-f\left(S S^{\prime}\right)
$$

is box totally dual integral. It was proved earlier by Cunningham [1977] and McDiarmid [1978] that for integer $f_{1}, f_{2}$ and $k$, the solution set of (47) has integer vertices, thus confirming a conjecture of Fulkerson [1971] on the blocking polyhedron of the common independent sets of size $k$ in two matroids. Indeed, (48) determines the set of all vectors $x$ for which there exists a vector $y \leq x$ in the intersection of the polymatroids corresponding to $f_{1}$ and $f_{2}$ with $y(S)=k$.

The box total dual integrality of (48) can be derived from that of (41) (Frank and Tardos [1982]).

Indeed, let $S^{*}$ be a disjoint copy of $S$. Let $\mathrm{C}_{1}=\left\{\left\{s, s^{*}\right\} \mid s \in S\right\}$ and $g_{1}\left(\left\{s, s^{*}\right\}\right)=1$ for all $s$ in $s$. Let $C_{2}=\left\{S_{1} \cup\left(S_{2}\right)^{\bullet} \mid\right.$ $\left.S_{2} \subseteq S_{1} \subseteq S\right\}$, and $g_{2}\left(S_{1} \cup\left(S_{2}\right)^{*}\right)=k-f_{1}\left(S S_{1}\right)-f_{2}\left(S_{2}\right)+\left|S_{2}\right|$. Then the system

$$
\begin{array}{ll}
x\left(\left\{s, s^{*}\right\}\right) \geq 1 & (s \in S), \\
x\left(S^{\prime}\right) \geq g_{2}\left(S^{\prime}\right) & \left(S^{\prime} \in C\right), \\
x\left(S \cup S^{*}\right)=|S| &
\end{array}
$$

is a special case of (41), and hence (49) is box totally dual integral. Therefore, also the system

$$
\begin{aligned}
& x\left(\left\{s, s^{*}\right\}\right)=1 \quad(s \in S), \\
& x\left(S \backslash\left(S_{1} \cup S_{2}\right)\right) \geq k-f_{1}\left(S_{1}\right)-f_{2}\left(S_{2}\right) \quad\left(S_{1}, S_{2} \subseteq S\right)
\end{aligned}
$$

is box totally dual integral, which implies that (48) is box totally dual integral.

XVII. KERNEL SYSTEMS (Frank [1979a]). Let $D=(V, A)$ be a directed graph, let $\mathbf{C}$ be an intersecting family on $V$, and let $g: \mathbf{C} \rightarrow \boldsymbol{R}$ be supermodular on intersecting pairs. Then the system

$$
\begin{aligned}
& x(a) \geq 0 \quad(a \in A), \\
& x\left(\delta^{-}\left(V^{\prime}\right)\right) \geq g\left(V^{\prime}\right) \quad\left(V^{\prime} \in \mathbf{C}\right)
\end{aligned}
$$

is box totally dual integral (Johnson [1975] considered the special case where $C=P(n\{r\})$ for some $r$ in $V)$. This may be derived from the 
box total dual integrality of (41) by the constructions given below in XVIII.

This model contains as special cases shortest paths (III), optimum arborescences (VII), and the matroid and polymatroid intersection (XII) (which is the special case where $D$ consists of pairwise disjoint arcs, and where $C=\left\{V^{\prime} \subseteq V \mid V^{\prime}\right.$ contains no tail or $V^{\prime}$ contains all heads $\}$ ). It also contains "blockers of common independent sets" (XVI) partly, namely if we add $x \geq 0$ to (48). For more applications, see Frank [1979a].

XVIII. CROSSING DIRECTED CUTS. Let $D=(V, A)$ be a directed graph, let $\mathbf{C}$ be a crossing family of subsets of $V$ such that $\delta^{+}\left(V^{\prime}\right)=\varnothing$ for all $V^{\prime}$ in $C$, and let $8: C \rightarrow R$ be supermodular on crossing pairs. Then the system

$$
x\left(8^{-}\left(V^{\prime}\right)\right) \geq g\left(V^{\prime}\right) \quad\left(V^{\prime} \in \mathbf{C}\right)
$$

is box totally dual integral (Edmonds and Giles [1977]). This can be derived from the box total integrality of (41) in a similar way as the Lucchesi-Younger theorem was derived in XIV.

Indeed, suppose we wish to show the total dual integrality of

$$
\begin{array}{lr}
c_{1}(a) \leq x(a) \leq c_{2}(a) & (a \in A), \\
x\left(\delta^{-}\left(V^{\prime}\right)\right) \geq 8\left(V^{\prime}\right) & \left(V^{\prime} \in C\right),
\end{array}
$$

for certain $c_{1}, c_{2}: A \rightarrow R$. Extend $D$ by adding an $\operatorname{arc} a^{\prime}=(w, v)$, for each arc $a=(v, w)$, thus obtaining $D^{\prime}=\left(V, A^{\prime}\right)$. Let $\mathbf{C}_{1}=\left\{\left\{a, a^{\prime}\right\} \mid a \in A\right\}, \delta_{1}\left(\left\{a, a^{\prime}\right\}\right)=c_{1}(a)+c_{2}(a)$,

$\mathbf{C}_{2}=\left\{H\left(V^{\prime}\right) \mid V^{\prime} \in C\right\}$, where $H\left(V^{\prime}\right)$ is the set of arcs of $D^{\prime}$ with head in $V^{\prime}$, and $g_{2}\left(H\left(V^{\prime}\right)\right)=g\left(V^{\prime}\right)+c_{1}\left(A\left(V^{\prime}\right)\right)+c_{2}\left(A\left(V^{\prime}\right)\right)$, where $A\left(V^{\prime}\right)$ denotes the set of arcs of $D$ contained in $V^{\prime}$. Then (41) has as special case:

$$
\begin{aligned}
& x\left(\left\{a, a^{\prime}\right\}\right) \geq g_{1}\left(\left\{a, a^{\prime}\right\}\right) \quad(a \in A), \\
& x\left(H\left(V^{\prime}\right)\right) \geq g_{2}\left(H\left(V^{\prime}\right)\right) \quad\left(V^{\prime} \in C\right), \\
& x\left(A \cup A^{\prime}\right)=c_{1}(A)+c_{2}(A) .
\end{aligned}
$$

The box total dual integrality of (53) then gives the total dual integrality of:

$$
\begin{array}{lc}
x\left(8_{\Lambda}^{-}\left(V^{\prime}\right)\right) \geq g\left(V^{\prime}\right) \quad\left(V^{\prime} \in \mathbf{C}\right), & (a \in A), \\
c_{1}(a) \leq x(a) \leq c_{2}(a) & \left.\left(a, a^{\prime}\right\}\right)=c_{1}(a)+c_{2}(a) \quad(a \in A),
\end{array}
$$


which implies the total dual integrality of (52).

Similarly, if $f: \mathbf{C} \rightarrow \boldsymbol{R}$ is submodular on crossing pairs, the system

$$
x\left(8^{-}\left(V^{\prime}\right)\right) \leq f\left(V^{\prime}\right) \quad\left(V^{\prime} \in \mathbf{C}\right)
$$

is box totally dual integral.

This scheme clearly contains Konig's theorems (I), the LucchesiYounger theorem (IX) and the box total dual integrality of (24) and (25), the matroid and polymatroid intersection (XII) (which is equivalent to the case where $D$ consists of pairwise disjoint arcs, and $\mathbf{C}=\left\{V^{\prime} \subseteq V \mid V^{\prime}\right.$ contains no tails or $U^{\prime}$ contains all heads $\}$ ), and therefore also the corollaries mentioned in XIII. It also contains the Kernel systems (XVII).

To see this, let $D=(V, A), C$ and $g$ be as in XVII. Let the digraph $D^{*}=\left(W, A^{*}\right)$ consist of pairwise disjoint $\operatorname{arcs}\left(v_{a}, w_{a}\right)$, for all $a$ in $A$. Let $C^{\prime}$ be the collection of all subsets $W^{\prime}$ of $W$ such that:

no arc of $D^{*}$ leaves $W^{\prime}$,

and there exists a set $V^{\prime}$ in $C$ such that for all $a=(v, w)$

in $A: w \in V^{\prime}$ iff $w_{a} \in W^{\prime}$, and $v \notin V^{\prime}$ implies $v_{a} \notin W^{\prime}$.

Let $g^{\circ}\left(W^{\prime}\right)=\max g\left(V^{\prime}\right)$, where the maximum ranges over all sets $V^{\prime}$ in $\mathbf{C}$ as described in (56). Then $\mathbf{C}^{*}$ is an intersecting family, and $\boldsymbol{g}^{\circ}$ is supermodular on intersecting pairs. Moreover, $\delta_{A^{*}}^{+}\left(W^{\prime}\right)=\varnothing$ for all $W^{\prime}$ in $\mathbf{C}^{*}$. Therefore, from (51), the system

$$
x\left(\delta_{A^{-}}^{-}\left(W^{\prime}\right)\right) \geq 8^{*}\left(W^{\prime}\right) \quad\left(W^{\prime} \in C^{*}\right)
$$

is box totally dual integral. Now one easily checks that for all $W^{\prime}$ in $C^{*}$ there is a $V^{\prime}$ in $C$ such that $8_{A^{-}}^{-}\left(W^{\prime}\right) \supseteq 8_{A}^{-}\left(V^{\prime}\right)$ and $8^{\circ}\left(W^{\prime} \leq g\left(V^{\prime}\right)\right.$ (identifying arcs of $D$ and those of $D^{\circ}$ in the obvious way). Conversely, for each $V^{\prime}$ in $C$ there is a $W^{\prime}$ in $C^{*}$ such that $8_{A^{\circ}}^{-}\left(W^{\prime}\right)=$ $8_{A}^{-}\left(V^{\prime}\right)$ and $g\left(V^{\prime}\right) \leq g^{\circ}\left(W^{\prime}\right)$. Hence the box total dual integrality of (57) implies that of (50).

Hence, also the consequences of the Kernel systems are contained in the present scheme.

Note that (55) directly contains (13) (Greene's union of chains (IV)). To see this, let $(P, \leq)$ be a partially ordered set. Let the digraph $D=(V, A)$ consist of pairwise disjoint $\operatorname{arcs}\left(p^{\prime}, p^{\prime \prime}\right)$, for $p$ in $P$. Let $\quad C=\left\{V^{\prime} \subseteq V \mid \quad p^{\prime} \in V^{\prime}=>p^{\prime \prime} \in V^{\prime}(p \in P)\right.$; $\left.p^{\prime \prime} \in V^{\prime}, p<q=>q^{\prime} \in V^{\prime}(p, q \in P)\right\}$. Then from (55) it follows that " $x\left(8^{-}\left(V^{\prime}\right)\right) \leq k \quad\left(V^{\prime} \in C\right)$ " is box totally dual integral, implying that (13) is totally dual integral. 
Moreover, (51) directly contains (48) (blockers of common independent sets (XVI)).

Indeed, let $S, f_{1}, f_{2}, k$ as in XVI be given. Let the digraph $D=(V, A)$ consist of pairwise disjoint arcs $\left(s^{\prime}, s^{\prime \prime}\right)$, for $s$ in $S$. Let $\mathrm{C}=\left\{V^{\prime} \subseteq V \mid s^{\prime} \in V^{\prime} \Rightarrow s^{\prime \prime} \in V^{\prime}(s \in S)\right\}$, and let $g\left(V^{\prime}\right)=$ $k-f_{1}\left(\left\{s \in S\left|s^{\prime \prime}\right| V^{\prime}\right\}\right)-f_{2}\left(\left\{s \in S \mid s^{\prime} \in V^{\prime}\right\}\right)$, for $V^{\prime} \in \mathbf{C}$. Then (51) is equivalent to (48).

XIX. CROSSING SYSTEMS ON DIRECTED GRAPHS I. Let $D=(V, A)$ be a directed graph, and let $C$ be a crossing family on $V$ such that if $V_{1}, V_{2}, V_{3}$ belong to $C$ with $V_{1} \subseteq V V_{2} \subseteq V_{3}$, then there is no arc of $D$ entering both $V_{1}$ and $V_{3}$. Let $g: C \rightarrow R$ be supermodular on crossing pairs. Then the system

$$
\begin{aligned}
& x(a) \geq 0 \quad(a \in A), \\
& x\left(\delta^{-}\left(V^{\prime}\right)\right) \geq g\left(V^{\prime}\right) \quad\left(V^{\prime} \in C\right)
\end{aligned}
$$

is box totally dual integral. I conjecture that this fact can be derived from the previous scheme, in a way similar to that of deriving "Optimum strong connectors" (X) from the Lucchesi-Younger theorem (IX).

The box total dual integrality of (58) implies that of all of the previous systems directly, with the exception of the network flows (II) and the orientations (XI).

If we add the condition that if $V_{1}$ and $V_{2}$ in $C$ are crossing then there is no arc in $D$ which enters $V_{1}$ and leaves $V_{2}$, then the following systems are box toally dual integral:

$$
\begin{array}{ll}
x\left(8^{-}\left(V^{\prime}\right)\right) \geq 8\left(V^{\prime}\right) & \left(V^{\prime} \in C\right), \\
x\left(8^{-}\left(V^{\prime}\right)\right) \leq f\left(V^{\prime}\right) & \left(V^{\prime} \in C\right),
\end{array}
$$

for $f: \mathbf{C} \rightarrow \mathbb{R}$ submodular on crossing pairs.

XX. CROSSING FAMILIES ON DIRECTED GRAPHS II: (Edmonds and Giles [1977]). Let $D=(V, A)$ be a directed graph, let $C$ be a crossing family of subsets of $V$, and let $f: C \rightarrow \mathbb{R}$ be submodular on crossing pairs. Then the system

$$
x\left(\delta^{-}\left(V^{\prime}\right)\right)-x\left(\delta^{+}\left(V^{\prime}\right)\right) \leq f\left(V^{\prime}\right) \quad\left(V^{\prime} \in \mathbf{C}\right)
$$

is box totally dual integral. This may be derived from scheme XIV (submodular functions on crossing families).

Indeed, let $c_{1}, c_{2}: A \rightarrow R$. Extend $D$ by new ares $a^{\prime}=(w, v)$ for each arc $a=(v, w)$, thus making the digraph $D^{\prime}=\left(V, A \cup A^{\prime}\right)$. Let $C_{1}=\left\{\left\{a, a^{\prime}\right\} \mid a \in A\right\}$, and $f_{1}\left(\left\{a, a^{\prime}\right\}\right)=c_{1}(a)+c_{2}(a)$. Let for 
each $V^{\prime}$ in $C, H\left(V^{\prime}\right)$ denote the set of arcs of $D^{\prime}$ with head in $V^{\prime}$. Let $\mathbf{C}_{2}=\left\{H\left(V^{\prime}\right) \mid V^{\prime} \in C\right\}$, and define $f_{2}\left(H\left(V^{\prime}\right)\right)=f\left(V^{\prime}\right)+$ $c_{1}\left(t\left(V^{\prime}\right)\right)+c_{2}\left(t\left(V^{\prime}\right)\right)$, where $t\left(V^{\prime}\right)$ denotes the set of arcs of $D$ with tail in $V^{\prime}$. Then the box total dual integrality of (41) gives that the system

$$
\begin{aligned}
& c_{1}(a) \leq x(a) \leq c_{2}(a) \quad(a \in A), \\
& x\left(\left\{a, a^{\prime}\right\}\right) \leq f_{1}\left(\left\{a, a^{\prime}\right\}\right) \quad(a \in A), \\
& x\left(H\left(V^{\prime}\right)\right) \leq f_{2}\left(H\left(V^{\prime}\right)\right) \quad\left(V^{\prime} \in C\right), \\
& x\left(A \cup A^{\prime}\right)=c_{1}(A)+c_{2}(A)
\end{aligned}
$$

is totally dual integral. This system is equivalent to:

$$
\begin{aligned}
& c_{1}(a) \leq x(a) \leq c_{2}(a) \quad(a \in A), \\
& x\left(\delta_{A}^{-}\left(V^{\prime}\right)\right)-x\left(\delta_{A}^{+}\left(V^{\prime}\right)\right) \leq f\left(V^{\prime}\right) \quad\left(V^{\prime} \in \mathbf{C}\right), \\
& x(a)+x\left(a^{\prime}\right)=c_{1}(a)+c_{2}\left(a^{\prime}\right) \quad(a \in A) .
\end{aligned}
$$

This implies that (59) is box totally dual integral.

Clearly, this immediately implies that, if $8: \mathbf{C} \rightarrow \boldsymbol{R}$ is supermodular on crossing pairs, the system

$$
x\left(\delta^{-}\left(V^{\prime}\right)\right)-x\left(8^{+}\left(V^{\prime}\right)\right) \geq g\left(V^{\prime}\right) \quad\left(V^{\prime} \in C\right)
$$

is box totally dual integral.

The box total integrality of (59) and (62) implies the total dual integrality in each of the schemes I-XVIII (maybe also XIX), either directly, or through the constructions described above.

Thus the generalized polymatroid scheme (XV), i.e., the box total dual integrality of (46), is included.

Indeed, let $S, \mathbf{B}_{1}, \mathbf{P}_{1}, \mathbf{B}_{2}, \mathbf{P}_{2}$ and $b_{1}, p_{1}, b_{2}, p_{2}$ be as in XV. Let the digraph $D=(V, A)$ consist of pairwise disjoint arcs $\left(v_{s}, w_{s}\right)$, for $s$ in $S$. Let $C$ consist of all subsets $V^{\prime}$ of $V$ such that:

$$
\begin{aligned}
V^{\prime} & =\left\{w_{s} \mid s \in S^{\prime}\right\} \quad \text { for some } S^{\prime} \text { in } \mathbf{B}_{1}, \\
\text { or } V^{\prime} & =h\left\{w_{s} \mid s \in S^{\prime}\right\} \quad \text { for some } S^{\prime} \text { in } P_{1}, \\
\text { or } V^{\prime} & =n\left\{v_{s} \mid s \in S^{\prime}\right\} \quad \text { for some } S^{\prime} \text { in } \mathbf{B}_{2}, \\
\text { or } V^{\prime} & =\left\{v_{s} \mid s \in S^{\prime}\right\} \quad \text { for some } S^{\prime} \text { in } P_{2} .
\end{aligned}
$$

Let $f\left(V^{\prime}\right):=b_{1}\left(S^{\prime}\right),:=p_{1}\left(S^{\prime}\right),:=b_{2}\left(S^{\prime}\right),:=p_{2}\left(S^{\prime}\right)$, respectively. Then $\mathbf{C}$ is a crossing family, and $f$ is submodular on crossing pairs. Moreover, the system (59) is equivalent to (46).

It also contains a result on orientations more general than (28) - 
see Frank [1980].

XXI. POLYMATROIDAL NETWORK FLOWS (Hassin [1978], Lawler and Martel [1982a,1982b]). Let $D=(V, A)$ be a directed graph, let, for each vertex $v$ of $D, C_{v}^{+}$and $C_{v}^{-}$be intersecting families on $\delta^{+}(v)$ and $\delta^{-}(v)$, respectively, and $f_{v}^{+}: \mathbf{C}_{v}^{+} \rightarrow \boldsymbol{R}$ and $f_{v}^{-}: \mathbf{C}_{v}^{-} \rightarrow \boldsymbol{R}$ be submodular on intersecting pairs. Then the system

$$
\begin{array}{lc}
x\left(\delta^{+}(v)\right)=x\left(\delta^{-}(v)\right) & (v \in V), \\
x\left(A^{\prime}\right) \leq f_{v}^{+}\left(A^{\prime}\right) & \left(v \in V, A^{\prime} \in C_{v}^{+}\right), \\
x\left(A^{\prime}\right) \leq f_{v}\left(A^{\prime}\right) & \left(v \in V, A^{\prime} \in C_{v}^{-}\right)
\end{array}
$$

is box totally dual integral. This may be derived from the EdmondsGiles scheme (cf. Frank [1982]).

Let the digraph $D^{\prime}=\left(V_{0}, A^{\prime}\right)$ consist of pairwise disjoint ares $a^{\prime}=\left(v_{a}, w_{a}\right)$, for each arc $a$ of $D$. Let $C$ consist of all subsets $V_{0}^{\prime}$ of $V_{0}$ such that there exists $a v$ in $V$ with:

(i) $V_{0}^{\prime}=\left\{u \in V_{0} \mid u\right.$ is head of some $a^{\prime}$ with $a$ in $\delta^{-}(v)$, or

$u$ is tail of some $a^{\prime}$ with $a$ in $\delta^{+}(v)$ \},

or (ii) there exists $A^{\prime}$ in $\mathrm{C}_{v}^{+}$with $V_{0}^{\prime}=\left\{u \in V_{0} \mid\right.$

$u$ is tail of somea' with $a$ in $\left.A^{\prime}\right\}$,

or (iii) there exists $A^{\prime}$ in $C_{v}^{-}$with $V_{0}^{\prime}=\left\{u \in V_{0} \mid\right.$

$u$ is not the head of any $a^{\prime}$ with $a$ in $\left.A^{\prime}\right\}$.

Define $f\left(V_{0}^{\prime}\right):=0,:=f_{v}^{+}\left(A^{\prime}\right),:=f_{\nu}\left(A^{\prime}\right)$, respectively. Then the box total dual integrality of (63) is equivalent to that of

$$
x\left(\delta_{A^{\prime}}^{-}\left(V_{0}^{\prime}\right)\right)-x\left(\delta_{A^{\prime}}^{+}\left(V_{0}^{\prime}\right)\right) \leq f\left(V_{0}^{\prime}\right) \quad\left(V_{0}^{\prime} \in C\right),
$$

which follows from XX.

In fact, the polymatroidal network flow model is equivalent to the Edmonds-Giles model, as the box total dual integrality of (59) follows from that of (63) (Lawler [1982]).

Indeed, let $D, C$ and $f$ as in $\mathbf{X X}$ be given. Let $C_{0}$ be the collection of all sets of the form $V_{1} \cap \cdots \cap V_{k}$, where $V_{1}, \ldots, V_{k}$ are sets in $C$ with $V_{l} \cup V_{j}=V$ for all $1 \leq i<j \leq k$. Then $C_{0}$ is an intersecting family. Define $f: C_{0} \rightarrow \boldsymbol{R}$ by $f_{0}\left(V^{\prime}\right)=\min \sum_{i=1}^{k} f\left(V_{i}\right)$, where the minimum ranges over all sets $V_{1}, \ldots, V_{k}$ as above with $V^{\prime}=V_{1} \cap \cdots \cap V_{k}$. Then $f_{0}$ is submodular on intersecting pairs (cf. Frank [1982]).

Now extend $D$ by a new point $r$ and new ares $(r, v)$ for $v$ in $V$, 
thus making the digraph $D_{0}=\left(V \cup\{r\}, A_{0}\right)$. Let $C_{r}^{+}=\left\{A^{\prime} \subseteq \delta_{A_{0}}^{+}(r) \mid\right.$ the set of heads of $A^{\prime}$ belongs to $C_{0}$. For $A^{\prime}$ in $\mathbf{C}_{r}^{+}$, let $f_{r}^{+}\left(A^{\prime}\right)=f\left(V^{\prime}\right)$, where $V^{\prime}$ is the set of heads of $A^{\prime}$. Then (63) implies that

$$
\begin{aligned}
& x\left(\delta_{A_{0}}^{+}(v)\right)=x\left(\delta_{A_{0}}^{-}(v)\right) \quad(v \in V), \\
& x\left(\delta_{A_{0}}^{+}(r)\right)=0, \\
& x\left(A^{\prime}\right) \leq f_{r}^{+}\left(A^{\prime}\right) \quad\left(A^{\prime} \in C_{r}^{+}\right)
\end{aligned}
$$

is box totally dual integral. But this is equivalent to (59) being box totally dual integral.

Many of the models described before are direct applications of the polymatroidal network flow model. Of course, through the constructions given above, each of the models I-XX is a special case.

The description of the polymatroidal network flows above is in terms of "independent circulations". There is an equivalent sourcesink model. That is, let $D=(V, A), C_{\nu}^{+}, C_{v}^{-}, f_{v}^{+}, f_{v}^{-}$be as above. Assume that the $f_{v}^{+}, f_{v}$ are nonnegative, and zero on $\varnothing$. Let $r$ and $s$ be two vertices of $D$. Then the maximum value of a flow $x$ from $r$ to $s$ satisfying

$$
\begin{array}{ll}
x\left(A^{\prime}\right) \leq f_{v}^{+}\left(A^{\prime}\right) & \left(v \in V, A^{\prime} \in C_{v}^{+}\right), \\
x\left(A^{\prime}\right) \leq f_{v}\left(A^{\prime}\right) & \left(v \in V, A^{\prime} \in C_{v}^{-}\right),
\end{array}
$$

(and, of course, $x \geq 0$ and $x\left(\delta^{-}(v)\right)=x\left(\delta^{+}(v)\right)$ for $\left.v \neq r, s\right)$, is equal to the minimum value of

$$
\sum_{v \in v}\left(f_{v}^{+}\left(A_{v}^{+}\right)+f_{v}\left(A_{v}^{-}\right)\right),
$$

where $A_{v}^{+} \in \mathrm{C}_{v}^{+}$and $A_{v}^{-} \in \mathrm{C}_{v}^{-}$such that $\cup_{\nu \in v}\left(A_{v}^{+} \cup A_{v}^{-}\right)$separates $r$ from $s$. This follows from the box total dual integrality of (63).

This contains directly the max-flow min-cut theorem (II) (by taking all $f_{v}^{+}$and $f_{v}$ to be modular) and polymatroid intersection (XII) (by taking for $D$ the graph with two points and several parallel arcs between them).

Hassin [1978] and Lawler [1982] extended the model to the case where on each $8^{+}(v)$ and $8^{-}(v)$ constraints of generalized polymatroid type (45) are defined. That is, for each $v$ in $V$ there are collections $\mathbf{B}_{v}^{+}, \mathbf{P}_{v}^{+}, \mathbf{B}_{v}^{-}, \mathbf{P}_{v}^{-}$and functions $b_{v}^{+}, p_{v}^{+}, b_{v}^{-}, p_{v}^{-}$on these collections satisfying (44). Then the system

$$
x\left(8^{+}(v)\right)=x\left(8^{-}(v)\right) \quad(v \in V),
$$




$$
\begin{array}{ll}
x\left(A^{\prime}\right) \leq b_{v}^{+}\left(A^{\prime}\right) & \left(v \in V, A^{\prime} \in B_{v}^{+}\right), \\
x\left(A^{\prime}\right) \geq p_{v}^{+}\left(A^{\prime}\right) & \left(v \in V, A^{\prime} \in P_{v}^{+}\right), \\
x\left(A^{\prime}\right) \leq b_{v}^{-}\left(A^{\prime}\right) & \left(v \in V, A^{\prime} \in B_{v}^{-}\right), \\
x\left(A^{\prime}\right) \geq p_{v}^{-}\left(A^{\prime}\right) & \left(v \in V, A^{\prime} \in P_{v}^{-}\right)
\end{array}
$$

is box totally dual integral. Again this may be seen to be equivalent to the Edmonds-Giles model by a similar construction as above.

Indeed, let again $D^{\prime}=\left(V_{0}, A^{\prime}\right)$ be the digraph consisting of pairwise disjoint $\operatorname{arcs} a^{\prime}=\left(v_{a}, w_{a}\right)$, for each arc $a$ of $D$. Now let $C$ consist of all subsets $V_{0}^{\prime}$ of $V_{0}$ such that there exists a $v$ in $V$ with:

$$
\begin{aligned}
& (i) V_{0}^{\prime}=h\left(8^{-}(v)\right) \cup t\left(8^{+}(v)\right), \\
& \text { or }(i i) V_{0}^{\prime}=t\left(A^{\prime \prime}\right) \quad \text { for some } A^{\prime \prime} \text { in } B_{v^{\prime}}^{+}, \\
& \text {or }(i i i) V_{0}^{\prime}=V_{0} v_{t}\left(A^{\prime \prime}\right) \quad \text { for some } A^{\prime \prime} \text { in } P_{v}^{+}, \\
& \text {or }(\text { iv }) V_{0}^{\prime}=V_{0} V_{h}\left(A^{\prime \prime}\right) \quad \text { for some } A^{\prime \prime} \text { in } B_{v}^{-}, \\
& \text {or }(v) V_{0}^{\prime}=h\left(A^{\prime \prime}\right) \quad \text { for some } A^{\prime \prime} \text { in } P_{v}^{-},
\end{aligned}
$$

where $t\left(A^{\prime}\right)=\left\{v_{a} \mid a \in A^{\prime}\right\}$ and $h\left(A^{\prime}\right)=\left\{w_{a} \mid a \in A^{\prime}\right\}$. Let $f\left(V_{0}^{\prime}\right):=0,:=b_{v}^{+}\left(A^{\prime}\right),:=p_{v}^{+}\left(A^{\prime}\right),:=b_{v}^{-}\left(A^{\prime}\right),:=p_{r}^{-}\left(A^{\prime}\right)$, respectively. Then the box total dual integrality of $(69)$ is equivalent to that of

$$
x\left(\delta_{A^{\prime}}^{-}\left(V_{0}^{\prime}\right)\right)-x\left(\delta_{A^{\prime}}^{+}\left(V_{0}^{\prime}\right)\right) \leq f\left(V_{0}^{\prime}\right) \quad\left(V_{0}^{\prime} \in \mathbf{C}\right),
$$

which follows from XX.

XXII. DISTRIBUTIVE LATTICES (Groflin and Hoffman [1982]). Let $L$ be a lattice, with minimal element $m$ and maximal element $M$, let $C \subseteq L$, let $r: C \rightarrow R$, let $n$ be a natural number, let, for $j=1, \ldots, n, h_{j}: L \rightarrow\{0,+1,-1\}$ such that for all $j:$

(i) $h_{j}(m)=h_{j}(M)=0$;

(ii) if $a, b, c \in L$ and $a \leq b \leq c$, then $\left|h_{j}(b)-h_{j}(a)\right| \leq 1$

and $\left|h_{j}(a)-h_{j}(b)+h_{j}(c)\right| \leq 1$;

(iii) $h_{j}(a)+h_{j}(b)=h_{j}(a \wedge b)+h_{j}(a \vee b)$ for all $a, b$ in $L$;

(iv) if $a, b \in C$ and $a \wedge b \neq m$ and $a \vee b \neq M$,

then $a \wedge b \in C$ and $a \vee b \in C$ and

$r(a)+r(b) \geq r(a \wedge b)+r(a \vee b)$.

Then the system 


$$
\sum_{j-1}^{n} h_{j}(a) x_{j} \leq r(a) \quad(a \in C)
$$

is box totally dual integral. Clearly, the Edmonds-Giles scheme follows by taking $L$ to be the collection of all subsets of the vertex set of a directed graph, and $h_{j}\left(V^{\prime}\right)=+1(-1$, respectively) iff arc numbered $j$ leaves (enters) $V^{\prime}$.

In fact, the model (72) is equivalent to the Edmonds-Giles model.

Indeed, let $L, m, M, C, r, n$ and $h_{j}$ as above. Let $V$ be the set of join-irreducible elements of $L$. Let, for each $c$ in $L, V_{c}:=\{b \in V \mid b \leq c\}$, let $\mathbf{C}=\left\{V_{c} \mid c \in C\right\}$, and let $f: \mathbf{C} \rightarrow \boldsymbol{R}$ be defined by $f\left(V_{c}\right)=r(c)$. So $C$ is a crossing family on $V$, and $f$ is submodular on crossing pairs. Observe that $V_{c} \wedge_{d}=V_{c} \cap V_{d}$ and $V_{c} v_{d}=V_{c} \cup V_{d}$.

Now choose some $j$. We may assume that $h_{j} \neq 0$, say $h_{j}(c)=+1$ for at least one $c$ in $L$. Since, by (72) (iii), the collection of elements of $L$ with $h_{j}(c)=1$ is closed under taking meets and joins, there are $c_{1} \leq c_{2}$ such that: $h_{j}(c)=1$ iff $c_{1} \leq c \leq c_{2}$. Note that $c_{1}$ is join-irreducible, since if $c_{1}=b^{\prime} \vee b^{\prime \prime}$ then $h_{j}\left(b^{\prime}\right)+h_{j}\left(b^{\prime \prime}\right)=$ $h_{j}\left(c_{1}\right)+h_{j}\left(b^{\prime} \wedge b^{\prime \prime}\right)$. If both $b^{\prime}<c_{1}$ and $b^{\prime \prime}<c_{1}$, then by (72) (ii), $h_{j}\left(b^{\prime}\right)=h_{j}\left(b^{\prime \prime}\right)=h_{j}\left(b^{\prime} \wedge b^{\prime \prime}\right)=0$.

There is a unique minimal element $c_{3}$ such that $c_{3} c_{2}$. For suppose there exists a second such element $c_{3}^{\prime}$. Then $0=h_{j}\left(c_{2} \vee c_{3}\right)+h_{j}\left(c_{2} \vee c_{3}^{\prime}\right)=h_{j}\left(c_{2} \vee c_{3} \vee c_{3}^{\prime}\right)+h_{j}\left(c_{2}\right)$, which implies that $h_{j}\left(c_{2} \vee c_{3} \vee c_{3}^{\prime}\right)=-1$, contradicting the fact that $c_{2} \vee c_{3} \vee c_{3}^{\prime} \geq c_{2}$. One similarly shows that $c_{3}$ is join-irreducible.

Now let $a$ be the $\operatorname{arc}\left(c_{1}, c_{3}\right)$. Then, for each $c$ in $L, h_{j}(c)=-1$ iff $a$ enters $V_{c}$, and $h_{j}(c)=+1$ iff $a$ leaves $V_{c}$. Indeed, $a$ leaves $V_{c}$, iff $c c_{3}$ and $c \geq c_{1}$, iff $c_{1} \leq c \leq c_{2}$, iff $h_{j}(c)=+1$. Moreover, suppose $a$ enters $V_{c}$, i.e., $c \geq c_{3}$ and $c c_{1}$. Then $h_{j}(c)=h_{j}\left(c \wedge c_{1}\right)+$ $h_{j}\left(c \vee c_{1}\right)-h_{j}\left(c_{1}\right)=-1$. Conversely, if $h_{j}(c)=-1$ then $c c_{1}$ and $c \quad c_{2}$ (by (72)(ii)), and hence $c \quad c_{1}$ and $c \geq c_{3}$, i.e., $a$ enters $V_{c}$.

This shows that the model (72) coincides with the Edmonds-Giles model.

Similarly, if the conditions (i) and (iv) are replaced by:

(i) $h_{j}(m)=0$

(iv) if $a, b \in C$ and $a \wedge b \neq m$, then $a \wedge b \in C$ and $a \vee b \in C$

$$
\text { and } r(a)+r(b) \leq r(a \wedge b)+r(a \vee b) \text {, }
$$

Then the system:

$$
x_{j} \geq 0 \quad(j=1, \ldots, n),
$$




$$
\sum_{j-1, h_{j}(a)-1}^{n} x_{j} \geq r(a) \quad(a \in C)
$$

is box totally dual integral. In the same way as above it follows that this variant is equivalent to the Kernel system model (XVII) (the proof of this fact is the same as above, as we may assume that $h_{j}(M)=0$ for all $j$ (otherwise add a new maximal element above the old one)).

Finally, if we take the conditions:

(i) $h_{j}(m)=0$;

(ii) as (72)(ii);

(iii) $h_{j} \geq 0$ and $h_{j}(a)+h_{j}(b) \leq h_{j}(a \wedge b)+h_{j}(a \vee b)$ for $a, b$ in $L$;

(iv) if $a, b \in C$ and $a b \neq m$, then $a \wedge b \in C$ and $a \vee b \in C$

$$
\text { and } r(a)+r(b) \geq r(a \wedge b)+r(a \vee b) \text {, }
$$

then the system:

$$
\begin{aligned}
& x_{j} \geq 0 \quad(j=1, \ldots, n), \\
& \sum, h_{j}(a) x_{j} \leq r(a) \quad(a \in C)
\end{aligned}
$$

is box totally dual integral. This model can be seen to be a special case of the Edmond-Giles model XVIII.

Indeed, let $D=(V, A)$ be a digraph with points $v_{1}, w_{1}, \ldots, v_{n}, w_{n}$, and ares $\left(v_{1}, w_{1}\right), \ldots,\left(v_{n}, w_{n}\right)$. Let $x_{j}=\wedge\left\{c \mid h_{j}(c)=1\right\}$ and $y_{j}=v\left\{c \mid h_{j}(c)=1\right\}$. So from (76) (iii) one has: $h_{j}(c)=1$ iff $x_{j} \leq c \leq y_{j}$. Define

$$
\mathrm{C}=\left\{V^{\prime} \subseteq V \mid \delta^{+}\left(V^{\prime}\right)=\varnothing \text { and there exists } c \text { in } \mathrm{C}\right. \text { with : }
$$

$$
\left.w_{j} \in V^{\prime}=>c \geq x_{j} \text {, and } v_{j} \notin V^{\prime}=>c \leq y_{j}\right\} \text {. }
$$

For $V^{\prime}$ in $\mathbf{C}$, define $f\left(V^{\prime}\right)=\min r(c)$, where the minimum ranges over all $c$ in $C$ with the properties described in (78). Then $C$ is a crossing family on $V$, and $f$ is submodular on crossing pairs. Hence, from the Edmonds-Giles scheme,

$$
x\left(8^{-}\left(V^{\prime}\right)\right) \leq f\left(V^{\prime}\right) \quad\left(V^{\prime} \in \mathbf{C}\right)
$$

is box totally dual integral. Now for each $V^{\prime}$ in $C$ there exists a $c$ in $C$ such that $h_{j}(c)=1$ if $\left(v_{j}, w_{j}\right)$ enters $V^{\prime}$, and $r(c)=f\left(V^{\prime}\right)$ (we could take the $c$ described in (78) minimizing $r(c)$, as $\left(v_{j}, w_{j}\right)$ enters $\left.V^{\prime}=>x_{j} \leq c \leq y_{j}=>h_{j}(c)=1\right)$. Conversely, for each $c$ in $\mathrm{C}$ there exists $V^{\prime}$ in $C$ such that: $\left(v_{j}, w_{j}\right)$ enters $V^{\prime}$ if $h_{j}(c)=1$, and $r(c) \geq f\left(V^{\prime}\right)$ (we could take $V^{\prime}=\left\{w_{j} \mid c \geq x_{j}\right\} \cup\left\{v_{j} \mid c y_{j}\right\}$, which set belongs to $C$, as if $\delta^{+}\left(V^{\prime}\right) \neq \varnothing$, there exist $v_{j} \in V^{\prime}, w_{j} \notin V^{\prime}$; hence $c y_{j}$ 
and $c x_{j}$; but then $1=h_{j}(c)+h_{j}\left(x_{j}\right) \leq h_{j}\left(c \wedge x_{j}\right)+h_{j}\left(c \vee x_{j}\right)=0$.

XXIII. LATTICE POLYHEDRA (Hoffman [1976,1978], Hoffman and Schwartz [1978], Groflin and Hoffman [1982]). Let $(L, \leq)$ be a partially ordered set, let $f: L \rightarrow \mathbb{R}$ and $h: L \rightarrow\{0,+1,-1\}^{n}$ be such that:

(i) if $a \leq b \leq c$ then $\left|h(a)_{j}-h(b)_{j}\right| \leq 1$ and

$$
\left|h(a)_{j}-h(b)_{j}+h(c)_{j}\right| \leq 1 \text { for } j=1, \ldots, n \text {; }
$$

(ii) if $a, b \in L$ with $a b a$, there exist $a \wedge b$

and $a \vee b$ in Lsuch thata $\wedge b<a$ and:

$$
\begin{aligned}
& f(a \wedge b)+f(a \vee b) \leq f(a)+f(b), \\
& h(a \wedge b)+h(a \vee b) \geq h(a)+h(b) .
\end{aligned}
$$

Then the system

$$
\begin{aligned}
& x_{j} \geq 0 \quad(j=1, \ldots, n), \\
& h(a) x \leq f(a) \quad(a \in L)
\end{aligned}
$$

is box totally dual integral. Moreover, if we strengthen the last inequality in (80)(ii) to:

$$
h(a \wedge b)+h(a \vee b)=h(a)+h(b),
$$

the system

$$
h(a) x \leq f(a) \quad(a \in L)
$$

is box totally dual integral. Clearly, the case of supermodular $f$ is equivalent.

These systems can be easily seen to contain Konig's theorems (I), the max-flow min-cut theorem (II), shortest paths (III), the results on matroids and polymatroids (XII) and their intersections (XIII), and on the "blockers of common independent sets" (XVI).

In this last case, let $S, f_{1}, f_{2}$ as in XVI. Let $L$ consist of all pairs $\left(S_{1}, S_{2}\right)$ with $S_{2} \subseteq S_{1} \subseteq S$, where $\left(S_{1}, S_{2}\right) \leqslant\left(S_{1}^{\prime}, S_{2}^{\prime}\right)$ iff $S_{1} \subseteq S_{1}^{\prime}$ and $S_{2} \subseteq S_{2}^{\prime}$. In fact, $L$ is a lattice. Let $\wedge$ and $\vee$ be the corresponding lattice operations. Define $h: L \rightarrow\{0,1\}^{S}$ by: $h\left(S_{1}, S_{2}\right)=$ the incidence vector of $S_{1} W_{2}$. Let $g\left(S_{1}, S_{2}\right)=k-f_{1}\left(S S_{1}\right) f_{2}\left(S_{2}\right)$. Then by $(83)$ the system

$$
\sum_{\text {, s }} h\left(S_{1}, S_{2}\right), x(s) \geq g\left(S_{1}, S_{2}\right) \quad\left(S_{2} \subseteq S_{1} \subseteq S\right)
$$

is box totally dual integral, which implies (48).

It also contains the results of Greene and Kleitman on partitions 
of partially ordered sets (IV) (Hoffman and Schwartz [1978]).

Indeed, let $(P \leqq)$ be a partially ordered set. Let $L$ be the set of all antichains in $P$, with $A \leq B$ iff $A^{+} \subseteq B^{l}$ (here $A^{+}$denotes the ideal in $P$ generated by $A$ ). Let $A \vee B$ be the collection of maximal elements of $A \cup B$. Let $A \wedge B=((A \cup B) \backslash(A \vee B)) \cup(A \cap B)$. Let $h: L \rightarrow\{0,1\}^{p}$, with $h(A)$ the incidence vector of $A$. Then (80)(i) and (82) are satisfied, and hence the system

$$
x(A) \leq k \quad(A \in L)
$$

is box totally dual integral, generalizing Greene's result on unions of chains (13). To obtain Greene and Kleitman's result on unions of antichains, define $h: L \rightarrow\{0,1\}^{P \cup\{r\}}$, where $r$ is a new element, by: $h(A)=$ the incidence vector of $A \cup\{r\}$; define $f: L \rightarrow R$ by $f(A)=|A|$. Then from the total dual integrality of (83) it follows that the system

$$
\begin{aligned}
& x(r) \geq 0 \\
& 0 \leq x(p) \leq 1 \quad(p \in P), \\
& x(A)+x(r) \geq|A| \quad(A \in L)
\end{aligned}
$$

is totally dual integral. Minimizing $x(P)+k x(r)$ over (86) gives Greene and Kleitman's result.

For more applications, see Hoffman and Schwartz [1978] and Groflin and Hoffman [1982].

XXIV. A MORE GENERAL FRAMEWORK (Schrijver [1982]). Let $C$ be a collection of subsets of a set $S$, let $n$ be a natural number, let $b, c \in \mathbb{R} \cup\{ \pm \infty\})^{n}$, let $f: \mathbf{C} \rightarrow \boldsymbol{R}$ and $h: \mathbf{C} \rightarrow\{0, \pm 1\}^{n}$ be such that:

(i) for all $j=1, \ldots, n$, if $S_{1}, S_{2}, S_{3}$ are pairwise noncrossing sets in $\mathbf{C}$,

there are $v, w$ in $S$ such that: $h_{j}\left(S_{l}\right)=+1$ iff $\operatorname{arc}(v, w)$

leaves $S_{i}$, and $h_{j}\left(S_{i}\right)+-1$ iff $\operatorname{arc}(v, w)$ enters $S_{i}$;

(ii) if $T$ and $U$ are crossing sets in $C$ there exist $T^{\prime}$ and $U^{\prime}$

in $C$ such that $T^{\prime} \subset T$ and

$f(T)+f(U)-f\left(T^{\prime}\right)-f\left(U^{\prime}\right) \geq\left(h(T)+h(U)-h\left(T^{\prime}\right)-h\left(U^{\prime}\right)\right) x$

for all $x$ with $b \leq x \leq c$.

Then the system

$$
\begin{aligned}
& b \leq x \leq c, \\
& h(T) x \leq f(T) \quad(T \in C)
\end{aligned}
$$


is box totally dual integral. Indeed, if we maximize a linear functional over (88), condition (87) (ii) gives that there exists an optimal dual solution whose active constraints correspond to a cross-free subfamily of C. Next condition (87)(i) implies that these constraints form a totally unimodular matrix, proving integer dual solutions if the objective function is integer.

This framework contains each of the schemes mentioned above as special case. Indeed, each of the schemes I-XXII follows by taking $S$ to be the set of vertices of some directed graph $D=(S, A)$, C some crossing family on $S, f: C \rightarrow \boldsymbol{R}$ submodular on crossing pairs, $h: \mathbf{C} \rightarrow\{0, \pm 1\}^{A}$ satisfying either (i) $h\left(V^{\prime}\right)_{a}=+1,-1,0$, respectively, corresponding to whether $a$ leaves $V^{\prime}$, enters $V^{\prime}$, or neither of them, respectively; or (ii) $h\left(V^{\prime}\right)=-1,0$, respectively, corresponding to whether $a$ enters $V^{\prime}$, or not, respectively. Under these conditions the schemes given in XIX and XX are the most general.

The framework of the lattice polyhedra (XIII) follows by taking for $\mathbf{C}$ the collection of all principal ideals in the partially ordered set (a principal ideal is a set $\{x \mid x \leq c\}$ for some $c$ ).

XXV. GRISHUHIN'S MODEL (Grishuhin [1981]). Let $L$ be a lattice, with minimal element $m$ and maximal element $M$, let $C \subseteq L, f: C \rightarrow \mathbb{R}$ and $h: C \rightarrow\{0, \pm 1\}^{n}$ such that:

(i) if $b, c \in C$ such that $b \wedge c \neq m$ and $b \vee c \neq M$ then

$$
\begin{aligned}
& b \wedge c, b \vee c \in C \text { and } f(b)+f(c) \geq f(b \wedge c)+f(b \vee c) \\
& \text { and } h(b)+h(c) \leq h(b \wedge c)+h(b \vee c) ;
\end{aligned}
$$

(ii) if $C^{\prime} \subseteq C$ is cross-free then the matrix $\left(h(c)_{j}: c \in C^{\prime} ; j=1, \ldots, n\right)$

is totally unimodular,

where a subset $C^{\prime}$ of $C$ is called cross-free if there are no $b, c$ in $C^{\prime}$ with $b \wedge c \neq m, b \vee c \neq M, b c$, and $c b$.

Then the system:

$$
\begin{aligned}
& x_{j} \geq 0 \quad(j=1, \ldots, n), \\
& \sum_{j=1}^{n} h(c) x_{j} \leq f(c) \quad(c \in C)
\end{aligned}
$$

is box totally dual integral. If we require $h$ to be modular (i.e., $\leq$ is replaced by $=$ in the second inequality of (89)(ii)), then we can delete the condition $x \geq 0$ in (90).

Grishuhin mentions two special cases. (1) $L$ is a lattice, $C \subseteq L, f: C \rightarrow \mathbb{R}, h: C \rightarrow\{0,1\}^{n}$, such that 
(i) if $b, c \in C$ then $b \wedge c, b \vee c \in C$ and $f(b)+f(c) \geq$ $f(b \wedge c)+f(b \vee c)$,

(ii) there are $b_{1}, c_{1}, \ldots, b_{n}, c_{n}$ in $L$ such that $h(c)_{j}=1$

if and only if $b_{j} \leq c \leq c_{j}$ for $j=1, \ldots, n$ and $c \in C$.

Indeed, after adding a new minimum $\hat{m}$ below the old minimum of $L$, and a new maximum $\hat{M}$ above the old maximum of $L$, we obtain the lattice $\hat{L}$. Then (91)(i) with respect to $L$ is equivalent to (89)(i) with respect to $\hat{L}$. Moreover, subsets of $C$ are cross-free in $\hat{L}$ iff they are chains in $L$. Hence (91)(ii) with respect to $L$ implies (89)(ii) with respect to $\hat{L}$.

Clearly, this special case is contained in the lattice polyhedra scheme (XXIII).

(2) $\mathbf{C}$ is a crossing family on a set $v, f: \mathbf{C} \rightarrow \boldsymbol{R}$ is submodular on crossing pairs, $R_{1}, S_{1}, \ldots, R_{n}, S_{n}$ are nonempty subsets of $V$, and $h: \mathbf{C} \rightarrow\{0, \pm 1\}^{n}$ satisfies:

$$
\begin{aligned}
& h\left(V^{\prime}\right)_{j}=+1 \text { iff } R_{j} \subseteq V^{\prime} \subseteq U s_{j}, \\
& h\left(V^{\prime}\right)_{j}=-1 \text { iff } S_{j} \subseteq V^{\prime} \subseteq V R_{j},
\end{aligned}
$$

for $j=1, \ldots, n$ and $V^{\prime} \in \mathbf{C}$. Then (89)(ii) is satisfied (for $L$ being the lattice family generated by C). However, the condition on $h$ in (89)(ii) generally is satisfied only if $\left|R_{1}\right|=\left|S_{1}\right|=\ldots=\left|R_{n}\right|=\left|S_{n}\right|=1$, i.e., in the Edmonds-Giles case (XX). (Grishuhin formulated this special case in the equivalent terms of a distributive lattice.)

\section{Optimum Cats and Connector-Collections, and Generalizations.}

We here survey some old and describe some new results yielding a type of min-max relation in a sense "polar" to those treated in the previous section. Now the programs ask for optimum objects of "cuttype", and for optimum collections of "connector type" objects.

We discuss the following schemes and interrelations. Notice that some of the results (viz. Menger's theorem, Greene and Kleitman's theorem on unions of antichains) fit both in the present list and into the previous one. The schemes LI-LXI do not presuppose a sub- or supermodular function.

LI. Edge-colourings in bipartite graphs (Konig [1916], Gupta [1967]).

LII. Menger's theorem and max-flow min-cut (Menger [1927], Ford and

Fulkerson [1956], Elias, Feinstein and Shannon [1956])): <= 
II.

LIII. Partitions of partially ordered sets (Dilworth [1950], Greene and Kleitman [1976]): $<=$ II, $<=$ IV .

LIV. The Hoffman-Schwartz chain model (Hoffman and Schwartz [1977]): $<=$ II, $=>$ LIII.

LV. Disjoint arborescences (Edmonds [1973]): => LII.

LVI. Disjoint bi-branchings (Schrijver [1982a]): = LI,LV.

LVII. Disjoint directed cut covers (Feofiloff and Younger [1982], Schrijver [1982a]): => LI.

LVIII. Disjoint strong connectors (Schrijver [1982a]): => LVI, LVII.

LIX. Crossing directed cuts: $<=$ XVIII, $=>$ LI, LIII.

LX. Kernel systems (Frank [1979a]): => LII,LV.

LXI. Crossing families (Schrijver [1983]): => LVII, LX.

LXII. Common colourings (Schrijver [1983a]): => LI.

LI. EDGE-COLOURINGS IN BIPARTITE GRAPHS. Let $G=(V, E)$ be a bipartite graph. Then the maximum degree of $G$ is equal to the minimum number of colours needed to colour the edges of $G$ such that no two edges of the same colour meet (Konig [1916]). The minimum degree of $G$ is equal to the maximum number of colours with which the edges of $G$ can be coloured such that in each point of $G$ all colours occur (Gupta [1967]). This implies that the following systems are totally dual integral:

$$
\begin{array}{ll}
x(e) \geq 0 & (e \in E), \\
x(M) \leq 1 & (M \subseteq E, M \text { matching }),
\end{array}
$$

and

$$
\begin{array}{ll}
x(e) \leq 0 & (e \in E), \\
x(C) \geq 1 & (C \subseteq E, C \text { coverings })
\end{array}
$$

(a covering is a set of edges covering $V$ ). This may be considered as "polar" to I. The systems (1) and (2) above, however, are not box totally dual integral (e.g., add " $x \leq \frac{1}{2}$ " to (1) or (2), and consider the graph obtained from $\boldsymbol{K}_{\mathbf{3}, 3}$ by deleting two disjoint edges).

De Werra [1976] showed more generally that if $G=(V, E)$ is a bipartite graph, and $k$ is a natural number, then the edges of $G$ can be coloured with $k$ colours such that in each point $v$ of $G$ there occur exactly $\min \{k$, degree $(v)\}$ colours. 
LII. MENGER'S THEOREM AND MAX-FLOW MIN-CUT. If $D=(V, A)$ is a directed graph and $r$ and $s$ are points of $D$, then the maximum number of pairwise arc-disjoint $r-s$-paths is equal to the minimum size of an $r-s$-cut (Menger [1927]). One derives from this the max-flow min-cut theorem (Ford and Fulkerson [1956], Elias, Feinstein and Shannon [1956]), which is equivalent to the total dual integrality of the following system:

$$
\begin{array}{ll}
x(a) \geq 1 & \\
x(P) \geq 1 & (P \subseteq A, P r-s \text {-path }) .
\end{array}
$$

This is polar to III (shortest paths), where $r-s-$ paths are exchanged with $r-s$-cuts. In scheme II the cuts were determined by singletons, which gives the max-flow min-cut theorem as a theorem of the first type.

In fact, for any number $k$, the system

$$
x(P) \geq k \quad(P \subseteq A, P r-s-\text { path })
$$

is box totally dual integral. This may be derived from the min-cost flow scheme II.

Indeed, add to any arc $a=(u, v)$ of $D$, an $\operatorname{arc} a^{\prime}=(v, u)$. Moreover, add the arc $(s, r)$. This makes the digraph $D^{\prime}=\left(V, A^{\prime}\right)$. Let $w: A \rightarrow Z$. Then the system

$$
\begin{aligned}
& y(a) \geq 0 \quad\left(a \in A^{\prime}\right), \\
& y\left(\delta_{A^{-}}^{-}(v)\right)-y\left(\delta_{A^{\prime}}^{+}(v)\right)=w\left(\delta_{A}^{+}(v)\right)-w\left(\delta_{A^{-}}^{-}(v)\right) \quad(v \in V) .
\end{aligned}
$$

is totally dual integral (cf. (3) of Section 2).

Hence, given $c, d: A \rightarrow \boldsymbol{R}$, the maximum of k. $y((s, r))+\sum_{a \in A}\left(c(a) y\left(a^{\prime}\right)-d(a) y(a)\right)$ over (5) has an integer optimum solution, which yields the integer optimum dual solution of the minimum of $\sum_{a \in A} w(a) x(a)$ over

$$
\begin{aligned}
& c \leq x \leq d, \\
& x(P) \geq k \quad(P \subseteq A, P r-s-\text { path }) .
\end{aligned}
$$

LIII. PARTIALLY ORDERED SETS. Let $(P, \checkmark)$ be a partially ordered set. The maximum size of an antichain is equal to the minimum number of chains needed to cover $P$ (Dilworth [1950]). This is equivalent to the total dual integrality of:

$$
\begin{array}{ll}
x(p) \geq 0 & (p \in P), \\
x(C) \leq 1 & (C \subseteq P, C \text { chain }) .
\end{array}
$$


This is again a result which fits in this list and in the previous one (cf. IV). tem

Greene and Kleitman [1976] showed more generally that the sys-

$$
\begin{array}{lc}
0 \leq x(p) \leq 1 & (p \in P), \\
x(C) \leq k & (C \subseteq P, C \text { chain })
\end{array}
$$

is totally dual integral, for each natural number $k$. In fact, the system

$$
x(C) \leq k \quad(C \subseteq P, C \text { chain })
$$

is box totally dual integral, for each number $k$, which may be derived again from the min-cost flow scheme II (cf. (14a) of Section 2).

LIV. THE HOFFMAN-SCHWARTZ CHAIN MODEL (Hoffman and Schwartz [1977]). Let $(P, S)$ be a partially ordered set, and let $C$ be the collection of chains in $P$. For $C, D$ in $C$, and $p$ in $C \cap D$, define the chain $(C, p, D)$ by

$$
(C, p, D)=\{q \in C \mid q \leq p\} \cup\{q \in D \mid q \geq p\} .
$$

Let $\boldsymbol{r}: \mathbf{C} \rightarrow \boldsymbol{Z}$ + satisfy:

(i) if $C \subseteq D \in C$, then $r(C) \leq r(D)$;

(ii) if $C, D \in \mathbf{C}, p \in C \cap D$, then $r(C)+r(D)=$

$$
r(C, p, D)+r(D, p, C)
$$

(such a function $r$ is called a switch function). Then the system

$$
\begin{array}{ll}
0 \leq x(p) \leq 1 & (p \in P), \\
x(C) \leq r(C) & (C \in C)
\end{array}
$$

is totally dual integral.

By induction on $|C|$ it is not difficult to see that

$$
r(C)=\sum_{i=1}^{i} r\left(\left\{p_{i}\right\}\right)+\sum_{i=1}^{s-1}\left(r\left(\left\{p_{i}, p_{i+1}\right\}\right)-r\left(\left\{p_{i}\right\}\right)-r\left(\left\{p_{i+1}\right\}\right)\right),
$$

if $C=\left\{p_{1}, \ldots, p_{n}\right\}$ with $p_{1}<p_{2}<\cdots<p_{n}(C \neq \varnothing)$. Therefore the following result is more general. Let $h:\{\{p, q\} \mid$ $p, q \in P, p \leq q\} \rightarrow \mathbb{R}$, and define $r: \mathbf{C} \rightarrow \mathbb{R}$ by:

$$
r(C):=\sum_{i=1}^{i} h\left(\left\{p_{i}\right\}\right)+\sum_{l=1}^{t-1} h\left(\left\{p_{i}, p_{i+1}\right\}\right),
$$

if $C=\left\{p_{1}, \ldots, p_{n}\right\}$ with $p_{1}<p_{2} \ldots<p_{n}$. Then the system

$$
x(C) \leq r(C) \quad(C \in C)
$$


is box totally dual integral. This may be derived from the min-cost flow scheme.

Indeed, make a digraph $D=(V, A)$ as follows. Let for each $p$ in $P$, points $p, p^{\prime}, p^{\prime \prime}$ be given. Moreover, let there be points $r$ and $s$. Let there be ares

$$
\begin{aligned}
& (r, p),\left(p, p^{\prime}\right),\left(p^{\prime}, p^{\prime \prime}\right),\left(p^{\prime \prime}, p^{\prime}\right),\left(p^{\prime \prime}, s\right) \quad(p \in P), \\
& \left(p^{\prime \prime}, q\right) \quad(p, q \in P, p<q), \\
& (s, r) .
\end{aligned}
$$

For $w: p \rightarrow \mathbb{R}$, consider the system:

$$
\begin{array}{lc}
y(a) \geq 0 & \left(a \in A, a \neq\left(p^{\prime}, p^{\prime \prime}\right) \text { for some } p \text { in } P\right), \\
y\left(p^{\prime}, p^{\prime \prime}\right) \geq w(p) & (p \in P), \\
y\left(\delta_{A}^{-}(v)\right)=y\left(\delta_{A}^{+}(v)\right) & (v \in V) .
\end{array}
$$

Given $c, d: P \rightarrow \mathbb{R}$, minimizing $\sum_{p \in P}\left(h(\{p\}) y\left(p, p^{\prime}\right)+d(p) y\left(p^{\prime \prime}, p^{\prime}\right)\right.$ $-c(p) y\left(p^{\prime}, p^{\prime \prime}\right)+\sum_{p<q} h(\{p, q\}) y\left(p^{\prime \prime}, q\right)$ over (17) yields integral dual variables to the linear program: maximize $\sum_{p \in p} w(p) x(p)$ subject to

$$
\begin{aligned}
& c \leq x \leq d \\
& x(C) \leq r(C) \quad(C \subseteq P, C \text { chain }) .
\end{aligned}
$$

A similar scheme can be designed for paths in directed graphs.

LV. DISJOINT ARBORESCENCES. Let $D=(V, A)$ be a digraph, and let $r \in V$. Then the minimum size of an $r$-cut is equal to the maximum number of pairwise disjoint $r$-arborescences (Edmonds [1973], cf. Lovász [1976]). [For definitions, see VII.] By splitting arcs to parallel ares a weighted version follows, which amounts to the total dual integrality of the system

$$
\begin{aligned}
& x(a) \geq 0 \quad(a \in A), \\
& x\left(A^{\prime}\right) \geq 1 \quad\left(A^{\prime} \subseteq A, A^{\prime} r\right. \text {-arborescence). }
\end{aligned}
$$

Menger's theorem (LII) is a direct consequence (indeed, to find pairwise disjoint $r-s$-paths, add arcs $(s, v)$ of high capacity, for all $v$ in $V$ (cf. Seymour [1977])).

Frank [1979a,1979b] and Vidyasankar [1978] derived from Edmonds' theorem a covering analogue: the minimum number of $r$ arborescences needed to cover $A$ is equal to the maximum among the numbers 


$$
\begin{aligned}
& \left|8^{-}(v)\right| \quad(v \in V), \\
& \left\lceil\left|<V^{\prime}>\right| /\left(\left|V^{\prime}\right|-1\right) \mid \quad\left(\varnothing \neq V^{\prime} \subseteq n\{r\}\right),\right.
\end{aligned}
$$

where $\left\langle V^{\prime}\right\rangle$ denotes the set of ares contained in $V^{\prime}$.

To derive this theorem from Edmonds' theorem, let $k$ be the maximum of (20), and add $k-\left|8^{-}(v)\right|$ arcs from $r$ to $v$, for each $v \neq r$. By Edmonds' theorem, the extended graph contains $k$ pairwise disjoint $r$-arborescences, which necessary cover all arcs. Removing the new ares gives a covering of the original graph.

I do not know a TDI-formulation of this result.

LVI. DISJOINT BI-BRANCHINGS. Let $D=(V, A)$ be a directed graph, and let $V$ be split into classes $U$ and $W$. Then the maximum number of pairwise disjoint bi-branchings is equal to the minimum size of an (U,W)-cut (Schrijver [1982a]). [For definitions, see VIII.] By making parallel ares one derives a weighted version, which is equivalent to the total dual integrality of the system

$$
\begin{aligned}
& x(a) \geq 0 \quad(a \in A), \\
& x\left(A^{\prime}\right) \geq 1 \quad\left(A^{\prime} \subseteq A, A^{\prime}\right. \text { bi-branching). }
\end{aligned}
$$

This result directly contains $\mathrm{LI}$ and $\mathrm{LV}$.

LVII. DISIOINT DIRECTED CUT COVERS. Let $D=(V, A)$ be an acyclic directed graph such that each pair of source and sink is connected by a directed path. Then the minimum size of a directed cut is equal to the maximum number of pairwise disjoint directed cut covers (Feofiloff and Younger [1982], Schrijver [1982a]). [For the definition of directed cut, see IX. A directed cut cover is a set of arcs intersecting all directed cuts, i.e., whose contraction makes the digraph strongly connected.] A more general weighted version also holds, which is equivalent to the total dual integrality of the system

$$
\begin{aligned}
& x(a) \geq 0 \quad(a \in A), \\
& x\left(A^{\prime}\right) \geq 1 \quad\left(A^{\prime} \subseteq A, A^{\prime} \text { directed cut cover }\right) .
\end{aligned}
$$

The condition of $D$ being acyclic is not essential (one could contract strong components without loss of generality). The condition that each source and sink is connected by a directed path cannot be deleted in general (cf. Schrijver [1980a]). However, we obtain the same result if we replace this last condition by the following alternative condition: $D$ arises from a directed tree by taking the transitive closure.

This scheme contains Gupta's edge-colouring theorem (LN).

Notice that it is equivalent to Dilworth's theorem (LII) that the 
maximum size of a directed cut is equal to the minimum number of arc sets $A_{1}, \ldots, A_{k}$ covering $A$ such that each $A_{i}$ intersects each directed cut at most once (see also $L I X)$.

LVIII. DISIOINT STRONG CONNECTORS. Let $D_{0}=\left(V, A_{0}\right)$ be an acyclic digraph such that each pair of source and sink of $D_{0}$ is connected by a directed path. Let $D=(V, A)$ be a second digraph. Then the maximum number of pairwise disjoint strong connectors (in $D$ for $D_{0}$ ) is equal to the minimum number of ares of $D$ entering a set $V^{\prime}$ of vertices, where $\varnothing \neq V^{\prime} \neq V$ and $\delta_{A_{0}}=\varnothing$ (Schrijver [1982a]). [For definitions, see X.] This may be derived from the previous scheme in a similar way as the optimum strong connector theorem in $\mathrm{X}$ was derived from the Lucchesi-Younger theorem (IX).

By adding parallel arcs a weighted version follows, which is equivalent to the total dual integrality of the system:

$$
\begin{aligned}
& x(a) \geq 0 \quad(a \in A), \\
& x\left(A^{\prime}\right) \geq 1 \quad\left(A^{\prime} \subseteq A, A^{\prime} \text { strong connector }\right) .
\end{aligned}
$$

This scheme contains Gupta's edge-colouring theorem (IN) (by taking $D_{0}$ to be a complete directed bipartite graph), Menger's theorem (LI) (by taking $A_{0}=\{(u, v) \mid u, v \in V, u=s$ or $v=r\}$ ), Edmonds' disjoint arborescence theorem (LV) (by taking $A_{0}=\{(v, r) \mid v \in V\}$ ), the disjoint bi-branching theorem (LVII) (by taking $\left.A_{0}=\{(u, v) \mid u \in U, v \in W\}\right)$, and the theorem on disjoint directed cut covers (LVII) (by taking $A \subseteq A_{0}^{-1}$ ).

LIX. CROSSING DIRECTED CUTS. Let $D=(V, A)$ be a directed graph, and let $C$ be a crossing family such that $\delta_{A}^{+}\left(V^{\prime}\right)=\varnothing$ for all $V^{\prime}$ in $C$. Then the maximum size of $\delta_{A}^{-}\left(V^{\prime}\right)$ for $V^{\prime}$ in $C$ is equal to the minimum number of arc sets $A_{1}, \ldots, A_{k}$ such that each $A_{l}$ enters such $V^{\prime}$ in $C$ at most once and such that $A_{1} \cup \ldots \cup A_{k}=A$. This follows from scheme XVIII combined with Lovász's perfect graph theorem (Lovász [1972]).

Indeed, (55) of Section 2 gives that the system

$$
\begin{array}{ll}
0 \leq x(a) \leq 1 & (a \in A), \\
x\left(8^{-}\left(V^{\prime}\right)\right) \leq 1 & \left(V^{\prime} \in C\right),
\end{array}
$$

is totally dual integral. Let $G$ be the undirected graph with point set $A$, where two arcs $a^{\prime}$ and $a^{\prime \prime}$ are adjacent, iff there is no set $V^{\prime}$ in $\mathbf{C}$ such that both $a^{\prime}$ and $a^{\prime \prime}$ enter $V^{\prime}$. Then $G$ is perfect. Hence also the complementary graph is perfect, which gives the desired result.

The result is equivalent to the total dual integrality of: 


$$
\begin{array}{ll}
0 \leq x(a) \leq 1 & (a \in A), \\
x\left(A^{\prime}\right) \leq 1 & \left(A^{\prime} \subseteq A, \delta_{A^{\prime}}\left(V^{\prime}\right) \leq 1 \text { for all } V^{\prime} \in C\right) .
\end{array}
$$

This contains as special cases Konig's edge-colouring theorem (LI) and Dilworth's theorem (LII). Call the perfect graphs obtained in this way Edmonds-Giles graphs. So the class of these graphs includes the comparability graphs and the complements of line graphs of bipartite graphs - see also Section 4.

Note that we may not replace the right hand side 1 of the second line of (25) by $k$ - see the counterexample given in LI.

By the remarks made in XIX (cf. (58b)), we can relax the condition " $\delta_{A}^{+}\left(V^{\prime}\right)=\varnothing$ for all $V^{\prime}$ in $C$ " to "if $V_{1}, V_{2}, V_{3}$ belong to $C$ and $V_{1} \subseteq V V_{2} \subseteq V_{3}$, then no arc of $D$ enters both $V_{1}$ and $V_{3}$, and if $V_{1}$ and $V_{2}$ are crossing then no arc of $D$ enters $V_{1}$ and leaves $V_{2}$ ".

LX. KERNEL SYSTEMS (Frank [1979a]). Let $D=(V, A)$ be a digraph, let $\mathbf{C}$ be an intersecting family on $V$, such that each $V^{\prime}$ in $\mathbf{C}$ is entered by at least $k$ arcs of $D$. Then $A$ can be split into classes $A_{1}, \ldots, A_{k}$ such that each $A_{t}$ enters each $V^{\prime} \in C$ at least once. This is equivalent to the total dual integrality of the system:

$$
\begin{array}{ll}
x(a) \geq 0 & (a \in A), \\
x\left(A^{\prime}\right) \geq 1 & \left(A^{\prime} \subseteq A, \delta_{A^{\prime}}^{-}\left(V^{\prime}\right) \geq 1 \text { for all } V^{\prime} \in C\right) .
\end{array}
$$

Menger's theorem (LI) and Edmonds' disjoint arborescence theorem (LV) are direct special cases.

LXI. CROSSING FAMIIIES (Schrijver [1983]). Let $D=(V, A)$ be a directed graph, and let $\mathbf{C}$ be a crossing family on $V$ such that

there are no $V_{1}, V_{2}, V_{3}, V_{4}, V_{5}$ in $C$ such that $V_{1} \subseteq V_{2} \cap V_{3}$,

$V_{2} \cup V_{3}=V, V_{3} \cup V_{4} \subseteq V_{5}, V_{3} \cap V_{4}=\varnothing$

Suppose that each set $V^{\prime} \in \mathbf{C}$ is entered by at least $k$ arcs of $D$. Then $A$ can be split into classes $A_{1}, \ldots, A_{k}$ such that each $A_{l}$ enters each $V^{\prime}$ in $C$ at least once. (In fact, (27) is a necessary and sufficient condition for this.) This is equivalent to the total dual integrality of the system

$$
\begin{aligned}
& x(a) \geq 0 \quad(a \in A), \\
& x\left(A^{\prime}\right) \geq 1 \quad\left(A^{\prime} \subseteq A, \delta_{A^{\prime}}^{-}\left(V^{\prime}\right) \geq 1 \text { for all } V^{\prime} \in C\right) .
\end{aligned}
$$

Special cases are Gupta's edge-colouring theorem (LD), Menger's theorem (LII), Edmonds' disjoint arborescence theorem (LV), the disjoint bi-branching theorem (LVI), the disjoint directed cut cover theorem (LVII), the disjoint strong connector theorem (LVII), and 
Frank's Kernel system theorem (LX).

The same result holds if we replace condition (27) by the alternative condition:

$C$ is cross - free, and there are no $V_{1}, V_{2}, V_{3}$ in $C$ with

$V_{1} \subseteq V_{1} \mid V_{2} \subseteq V_{3}$ for which there exists an arc of $D$

entering both $V_{1}$ and $V_{3}$.

This follows from the fact that (29) gives a totally unimodular constraint matrix. Is there a common generalization for (27) and (29)?

The only generalization of this type involving sub- or supermodular functions I know, is the following, which could be considered as "polar" to the polymatroid intersection (XII).

LXII. COMMON COLOURINGS (Schrijver [1983a]). Let $C_{1}$ and $C_{2}$ be intersecting families on a set $S$, and let $8_{1}: C_{1} \rightarrow \boldsymbol{Z}$ and $8_{2}: C_{2} \rightarrow Z$ be supermodular on intersecting pairs, such that $g_{i}\left(S^{\prime}\right) \leq\left|S^{\prime}\right|$ for $i=1,2$ and $S^{\prime} \in \mathbf{C}_{i}$. Then the minimum number of colours needed to colour $S$ such that $S^{\prime}$ contains at least $g_{i}\left(S^{\prime}\right)$ colours $\left(i=1,2 ; S^{\prime} \in C_{i}\right)$ is equal to the maximum of $g_{i}\left(S^{\prime}\right)\left(i=1,2 ; S^{\prime} \in C_{i}\right)$.

I do not know a formulation in terms of total dual integrality of this result. It contains as special cases Konig's and Gupta's edgecolouring theorems (LI), and the more general result of De Werra (LI).

The following more general result might be true. Let $\mathbf{C}$ be a crossing family on $V$, such that there are no $V_{1}, V_{2}, V_{3}$ in $C$ with $V_{1} \subseteq \eta V_{2} \subseteq V_{3}$. Let $g: C \rightarrow \boldsymbol{Z}$ be supermodular on crossing pairs, and let $D=(V, A)$ be a digraph such that $\left|\delta_{A}^{-}\left(V^{\prime}\right)\right| \geq g\left(V^{\prime}\right)$ for all $V^{\prime}$ in C. Let $k=\max _{V^{\prime} \in c g}\left(V^{\prime}\right)$. Then $A$ can be split into classes $A_{1}, \ldots, A_{k}$ such that each set $V^{\prime}$ in $C$ is entered by at least $g\left(V^{\prime}\right)$ of the $A_{i}$ ??? This would generalize most of the results of this Section.

Note that the following is not true in general: if $D=(V, A)$ is a digraph, $\mathbf{C}$ is a lattice family, $\boldsymbol{g}: \mathbf{C}-\boldsymbol{Z}$ is supermodular, $k$ is a natural number such that $\left|\delta_{A}^{-}\left(V^{\prime}\right)\right| \geq k \cdot g\left(V^{\prime}\right)$ for all $V^{\prime}$ in $C$, then $A$ can be split into $k$ classes $A_{1}, \ldots, A_{k}$ such that $\left|\delta_{A_{i}}^{-}\left(V^{\prime}\right)\right| \geq g\left(V^{\prime}\right)$ for $V^{\prime}$ in $C$ and $i=1, \ldots, k$. Indeed, for $D$ consisting of pairwise disjoint arcs, and $\mathbf{C}=\left\{V^{\prime} \subseteq V \mid V^{\prime}\right.$ contains no tails, or $V^{\prime}$ contains all heads $\}$, this would imply the false result that if $S$ can be split into $k$ bases of matroid $M_{1}$, and also into $k$ bases of matroid $M_{2}$, then $S$ can be split into $k$ common bases. 


\section{Some Remarts on the Algorithmic Complexity.}

So far we have neglected the algorithmic aspects of the several frameworks - we refer to the papers mentioned in Section 1. In these papers the sub- and supermodular functions are assumed to be given by an "oracle". This seems necessary as there can exist generally more than exponentially many such functions.

If the model involves a crossing family $C$ on a finite set $S$, it is possible to represent this family in size polynomially bounded by $|S|$, as it suffices to specify:

(i) whether $\varnothing \in \mathbf{C}$, and whether $S \in \mathbf{C}$;

(ii) for all $s_{1}, s_{2}, s_{3}, s_{4}$ in $S$, whether there exists an $S^{\prime}$ in $C$ with

$$
s_{1}, s_{2} \in S^{\prime} \text { and } s_{3}, s_{4} \neq S^{\prime} \text {. }
$$

Indeed, it is easy to see that a nonempty proper subset $S^{\prime \prime}$ of $S$ belongs to $C$, if and only if for all $s_{1}, s_{2} \in S^{\prime \prime}$ and for all $s_{3}, s_{4} \in S S^{\prime \prime}$, question (1)(ii) is confirmed.

So one may associate with a crossing family $\mathbf{C}$ on $S$ a relation between members of the set $S^{2}:=\left\{\left\{s_{1}, s_{2}\right\} \mid s_{1}, s_{2} \in S\right\}$, by:

$$
\begin{aligned}
& \left\{s_{1}, s_{2}\right\} \rightarrow\left\{s_{3}, s_{4}\right\} \text { iff for all } S^{\prime} \in C:\left(s_{1}, s_{2} \in S^{\prime}\right. \text { implies } \\
& \left.s_{3} \in S^{\prime} \text { or } s_{4} \in S^{\prime}\right)
\end{aligned}
$$

$\left(s_{1}, s_{2}, s_{3}, s_{4}\right.$ need not be distinct). Hence the relation $\rightarrow$ determines $\mathbf{C}$ (except for the inessential elements $\varnothing$ and $S$ ). It can be shown that a relation $\rightarrow$ on $S^{2}$ belongs to some crossing family $C$, if and only if it satisfies the following axioms, for all $s_{1}, s_{2}, s_{3}, s_{4}, s_{5}$ in $S$ :

(i) $\left\{s_{1}, s_{2}\right\} \rightarrow\left\{s_{2}, s_{3}\right\}$,

(ii) if $\left\{s_{1}, s_{2}\right\} \rightarrow\left\{s_{3}, s_{4}\right\}$ and $\left\{s_{2}, s_{3}\right\} \rightarrow\left\{s_{4}, s_{3}\right\}$ then

$$
\left\{s_{1}, s_{2}\right\} \rightarrow\left\{s_{4}, s_{s}\right\} \text {, }
$$

(iii) if $\left\{s_{1}, s_{2}\right\} \rightarrow\left\{s_{3}, s_{4}\right\}$ then $\left\{s_{1}, s_{2}\right\} \rightarrow\left\{s_{3}, s_{s}\right\}$ or

$$
\left\{s_{1}, s_{2}\right\} \rightarrow\left\{s_{4}, s_{5}\right\} \text {, }
$$

(iv) if $\left\{s_{2}, s_{3}\right\} \rightarrow\left\{s_{4}, s_{3}\right\}$ then $\left\{s_{1}, s_{2}\right\} \rightarrow\left\{s_{4}, s_{3}\right\}$ or

$$
\left\{s_{1}, s_{3}\right\} \rightarrow\left\{s_{4}, s_{s}\right\} \text {. }
$$

This also shows that the class of (perfect) Edmonds-Giles graphs, described in scheme LIX is constructive. In fact, this class of graph can be described as follows. Let $S$ be a finite set, and let $\rightarrow$ be a relation on $S^{(2)}:=\left\{\left\{s_{1}, s_{2}\right\} \mid s_{1}, s_{2} \in S, s_{1} \neq s_{2}\right\}$ satisfying the conditions 
(3) (ii) (iii) (iv). Let $E$ be the set of pairs $\left\{s_{1}, s_{2}\right\}$ with $\left\{s_{1}, s_{2}\right\} \rightarrow\left\{s_{1}, s_{2}\right\}$. Then $(S, E)$ is an Edmonds-Giles graph, and each Edmonds-Giles graph arises in this way. This class contains the comparability graphs and the complements of line graphs of bipartite graphs. If $(S, S)$ is a partially ordered set, and we define

$$
\left\{s_{1}, s_{2}\right\}-\left\{s_{3}, s_{4}\right\} \text { iff } s_{i}<s_{j} \text { for some } i=1,2 \text { and } j=3,4 \text {, }
$$

then - satisfies (3) (ii) (iii) (iv), and we obtain the corresponding comparability graph. If $S$ is the edge set of a bipartite graph, say with vertices coloured red and blue, and if we define

$\left\{s_{1}, s_{2}\right\} \rightarrow\left\{s_{3}, s_{4}\right\}$ iff $s_{1}$ and $s_{2}$ have no red vertex in common and

$s_{3}$ and $s_{4}$ have no blue vertex in common,

then $\rightarrow$ satisfies (3)(ii) (iii) (iv), and we obtain the complement of the line graph of the bipartite graph.

So the problem of testing whether a given graph is an EdmondsGiles graph belongs to the class NP. Does this problem also belong to $P$, i.e., can the property of being an Edmonds-Giles graph be tested in polynomial time?

It may be derived with the ellipsoid method (cf. Grotschel, Lovasz and Schrijver [1981]) that the optimization problems corresponding to the frameworks I-XXII and LI-LXI are polynomially solvable, assuming that the sub- or supermodular functions in question are given by an oracle. We do not know to what extent the optimization problems corresponding to the other models are polynomially solvable.

\section{Acknowledgements}

I thank Dr. Andras Frank and an unknown referee for their helpful comments.

\section{References}

[1977] W.H. Cunningham, An unbounded matroid intersection polyhedron, Linear Algebra and Its Appl. 16 (1977) 209-215.

[1982] W.H. Cunningham and A. Frank, A primal-dual algorithm for submodular flows, Report 82224-OR, Institur fur Operations Research, Bonn, 1982 (to appear in Mathematics of Operations Research).

[1950] R.P. Dilworth, A decomposition theorem for partially ordered sets, Ann. of Math. $S 1$ (1950) 161-166.

[1976] F.D.J. Dunstan, Matroids and submodular functions, Quart. J. Math. Oxford (2) 27 (1976) 339-348.

[1967] J. Edmonds, Optimum branchings, J. Res. Nat. Bur. Standards Section B 71 (1967) 233-240. 
[1970] J. Edmonds, Submodular functions, matroids, and cerrain polyhedra, in: "Combinatorial structures and their applications" (R. Guy, et al., eds), Gordon and Breach, New York, 1970, 69-87.

[1971] J. Edmonds, Matroids and the greedy algorithm, Math. Programming 1 (1971) 127-136.

[1973] J. Edmonds, Edge-disjoint branchings, in: Combinatorial algorithms" (R. Rusin, ed. ), Academic Press, New York, 1973, 91-96.

[1979] J. Edmonds, Matroid intersection, Annals of Discrete Math. 4 (1979) 39-49.

[1977] J. Edmonds and R. Giles, A min-max relation for submodular functions on graphs, Annals of Discrete Math. 1 (1977) 185-204.

[1956] P. Elias, A. Feinstein and C.E. Shannon, A note on the maximum flow through a network, IRE Trans. Information Theory IT 2 (1956) 117-119.

[1982] P. Feofiloff and D.H. Younger, Directed cur transversal packing for source-sink connected graphs, preprint, 1982.

[1956] L.R. Ford and D.R. Fulkerson, Maximum flow through a network, Canad. $J$. Math. 8 (1956) 399-404.

[1958] L.R. Ford and D.R. Fulkerson, Network flow and systems of distinct representatives, Canad. J. Math. 10 (1958) 78-84.

[1962] L.R. Ford and D.R. Fulkerson, Fows in networks, Princeton Univ. Press, Princeton, N.J., 1962.

[1979a] A. Frank, Kernel systems of directed graphs, Acta Sci. Math. (Szeged) 41 (1979) 63-76.

[1979b] A. Frank, Covering branchings, Acta Sci. Math. (Szeged) 41 (1979) 77-81.

[1980] A. Frank, On the orientation of graphs, J. Combinatorial Theory (B) 28 (1980) 251-261.

[1980a] A. Frank, On chain and antichain families of a partially ordered set, J. Combinatorial Theory (B) 29 (1980) 176-184.

[1981] A. Frank, How to make a digraph strongly connected, Combinatorica 1 (1981) 145-153.

[1981a] A. Frank, A weighted matroid intersection theorem, J. of Algorithms 2 (1981) 328-336.

[1981b] A Frank, Generalized polymatroids, Report 81206-OR, Institut für Operations Research, Bonn, 1981 (to appear in Proceedings Sixth Hungarian Combinatorial Collo quium (Eger, 1981)).

[1981c] A. Frank, Finding feasible vectors in Edmonds-Giles polyhedra, Report 81205 OR, Institur für Operations Research, Bonn, 1981 (to appear in Journal of Combinatorial Theory (B)).

[1982] A. Frank, An algorithm for submodular functions on graphs, Annals of Discrete Math. 16 (1982) 97-120.

[1981] A. Frank and E. Tardos, Matroids from crossing families, Report 82210-OR, Institur für Operations Research, Bonn, 1982 (to appear in Proceedings Sixth Hungarian Combinatorial Colloquium (Eger, 1981)).

[1982] A. Frank and E. Tardos, An algorithm for the unbounded matroid intersection polyhedron, Report 82252-OR, Institut für Operations Research, Bonn, 1982 (to appear in Proceedings Colloquium on Matroids (Szeged, 1982)). 
[1978] S. Fujishige, Algorithms for solving the independent flow problems, J. Oper. Res. Soc. Japan 21 (1978) 189-203.

[1956] D.R. Fulkerson, Note on Dilworth's decomposition theorem for partially ordered sets, Proc. Amer. Math. Soc. 7 (1956) 701-702.

[1971] D.R. Fulkerson, Blocking and anti-blocking pairs of polyhedra, Math. Programming 1 (1971) 168-194.

[1974] D.R. Fulkerson, Packing rooted directed cuts in a weighred directed graph, Math. Programming 6 (1974) 1-13.

[1979] F.R. Giles and W.R. Pulleyblank, Total dual integrality and integer polyhedra, Linear Algebra and Its Appl. 25 (1979) 191-196.

[1976] C. Greene, Some partitions associated with a partially ordered set, J. Combinatorial Theory (A) 20 (1976) 69-79.

[1976] C. Greene and D.J. Kleiman, The structure of Sperner $k$-families, J. Combinatorial Theory (A) 20 (1976) 41-68.

[1981] V.P. Grishuhin, Polyhedra related to a lattice, Math. Programming 21 (1981) 70 89.

[1981] H. Groflin and A.J. Hoffman, On matroid intersections, Combinatorica 1 (1981) 43-47.

[1982] H. Groflin and A.J. Hoffman, Lattice polyhedra II: generalization, constructions and examples, preprint.

[1981] M. Grotschel, L. Lovász and A Schrijver, The ellipsoid method and its consequences in combinatorial optimization, Combinatorica 1 (1981) 169-197.

[1967] R.P. Gupta, A decomposition theorem for bipartite graphs, in: "Theory of graphs" (P. Rosenstiehl, ed.), Gordon and Breach, New York, 1967, 135-138.

[1978] R. Hassin, On network flows, Ph.D. Dissertation, Yale University, 1978.

[1960] A.J. Hoffman, Some recent applications of the theory of linear inequalities to extremal combinatorial analysis, in: "Combinatorial analysis" (R.E. Bellman and M. Hall Jr., eds. ), Amer. Math. Soc., Providence, R. I., 1960, 113-127.

[1974] A.J. Hoffman, A generalization of max-flow min-cut, Math. Programming 6 (1974) 352-359.

[1976] A.J. Hoffman, On lattice polyhedra II, constructions and examples, Report RC 6268, IBM Thomas J. Watson Res. Center, Yorktown Heights, N. Y., 1976.

[1978] A.J. Hoffman, On lattice polyhectra III: Blockers and anti-blockers of lattice clutters, Math. Progr. Study 8 (1978) 197-207.

[1977] A.J. Hoffman and D.E. Schwart, On partitions of partially ordered sets, J. Combinatorial Theory (B) 23 (1977) 3-13.

[1978] A.J. Hoffman and D.E. Schwartz, On latice polyhedra, in: "Combinatorics" (A. Hajnal and V.T. Sos, eds. ), North-Holland, Amsterdam, 1978, pp. 593-598.

[1975] E.L. Johnson, On cut-set integer polyhedra, Cahiers du Centre Oper. Rech. (Actes Journées Franco-Belges) 17 (1975) 235-251.

[1979] A. V. Karzanov, On the minimal number of ares of a directed graph meeting all its directed cutsets (abstract), Graph Theory Newsletters 8 (1979).

[1916] D. König, Graphen und ihre Anwendung auf Determinantentheorie und Mengenlehre, Math. Ann. 77 (1916) 453-465.

[1931] D. Kơnig, Graphok es matrixok, Mat. Fiz. Lapok 38 (1931) 116-119. 
[1932] D. Kơnig, Uber trennende Knotenpunkte in Graphen (nebst Anwendungen auf Determinanten und Matrizen), Acta Lit. Sci. Sect. Sci. Math. (Szeged) 6 (1932-1934) 155-179.

[1975] E.L. Lawler, Matroid intersection algorithms, Math. Programming 9 (1975) 31 56.

[1982] E.L. Lawler, Generalizations of the polymatroidal network flow, Report BW 158, Mathematisch Centrum, Amsterdam, 1982.

[1982a] E.L. Lawler and C.U. Martel, Computing maximal "polymatroidal" network flows, Math. of Oper. Research 7 (1982) 334-347.

[1982b] E.L. Lawler and C.U. Marte, Flow network formulations of polymatroid optimization problems, Arrals of Discrete Math. 16 (1982).

[1972] L. Lovasz, Normal hypergraphs and the perfect graph conjecture, Discrete Math. 2 (1972) 253-267.

[1976] L. Lovász, On two minimax theorems in graph theory, J. Combinatorial Theory (B) 21 (1976) 96-103.

[1977] L. Lovász, Flats in matroids and geometric lattices, in: "Combinatorial surveys" (P.J. Cameron, ed.), Academic Press, London, 1977, 45-86.

[1976] C.L. Lucchesi, A minimax equality for directed graphs, Doctoral Thesis, Univ. of Waterloo, Waterloo, Ont., 1976.

[1978] C.L. Lucchesi and D.H. Younger, A minimax relation for directed graphs, J. London Math. Soc. (2) 17 (1978) 369-374.

[1978] C. McDiarmid, Blocking, anti-blocking, and pairs of matroids and polymatroids, J. Combinatorial Theory (B) 25 (1978) 313-325.

[1927] K. Menger, Zur allgemeinen Kurventheorie, Fund. Math. 10 (1927) 96-115.

[1966] L. Mirsky and H. Perfect, Systems of representatives, J. Math. Analysis Appl. 15 (1966) 520-568.

[1969] C.St.J.A. Nash-Williams, Well-balanced orientations of finite graphs and unobtrusive odd-vertex-pairings, in: "Recent progress in combinatorics" (W.T. Turte, ed.), Academic Press, New York, 1969, 133-149.

[1980] P. Schorasleben, Ganzzahlige Polymatroid-Intersektions-Algorithmen, Ph.D. Thesis, Eidgenossischen Technischen Hochschule, Zürich, 1980.

[1980] A. Schrijver, A counterexample to a conjecture of Edmonds and Giles, Discrete Math. 32 (1980) 213-214.

[1981] A. Schrijver, On total dual integrality, Linear Algebra and Its Appl. 38 (1981) $27-32$.

[1982] A Schrijver, Proving total dual integrality with cross-free families - a general framework, Report AE 5/82, University Amsterdam, 1982. (to appear in Math. Programming).

[1982a] A Schrijver, Min-max relations for directed graphs, Annals of Discrete Math. 16 (1982) 261-280.

[1983] A. Schrijver, Packing and covering of crossing families of cuts, Report AE 1/83, University Amsterdam, 1983. (to appear in J. Combinatorlal Theory (B)).

[1983a] A. Schrijver, Supermoctular colourings, submitted to Proceedings Colloquium on Matroids (Szeged, 1982).

[1977] P.D. Seymour, The matroids with the max-flow min-cur property, $J$. 
Combinatorial Theory (B) 23 (1977) 189-222.

[1978] K. Vidyasankar, Covering the edge set of a directed graph with trees, Discrete Maxh. 24 (1978) 79-85.

[1976] D. de Werra, Some remarks on good colorations, J. Combinatorial Theory $(B) 21$ (1976) $57-64$. 